DOUGLAS RENE ROCHA SILVA

\title{
Poluição atmosférica como fator de risco para o déficit cognitivo no Estudo Longitudinal da Saúde do Adulto - ELSA Brasil
}

\author{
Dissertação apresentada à Faculdade de Medicina da \\ Universidade de São Paulo para obtenção do título de \\ Mestre em Ciências \\ Programa de Saúde Coletiva \\ Orientador: Prof. Nelson da Cruz Gouveia \\ Co-orientadora: Claudia Kimie Suemoto
}

(Versão corrígida Resolução CoPGr 6018/11, de 1 de novembro de 2011. A versão original está disponível na Biblioteca da FMUSP). 
DOUGLAS RENE ROCHA SILVA

Poluição atmosférica como fator de risco para o déficit cognitivo no Estudo

Longitudinal da Saúde do Adulto - ELSA Brasil

Dissertação apresentada à Faculdade de Medicina da Universidade de São Paulo para obtenção do título de Mestre em Ciências

Programa de Saúde Coletiva

Orientador: Prof. Nelson da Cruz Gouveia

Co-orientadora: Claudia Kimie Suemoto

São Paulo

2020 


\section{Dados Internacionais de Catalogação na Publicaçăo (CIP)}

Preparada pela Bíblioteca da

Faculdade de Medicina da Universidade de Săo Paulo

Creproduçăo autorizada pelo autor

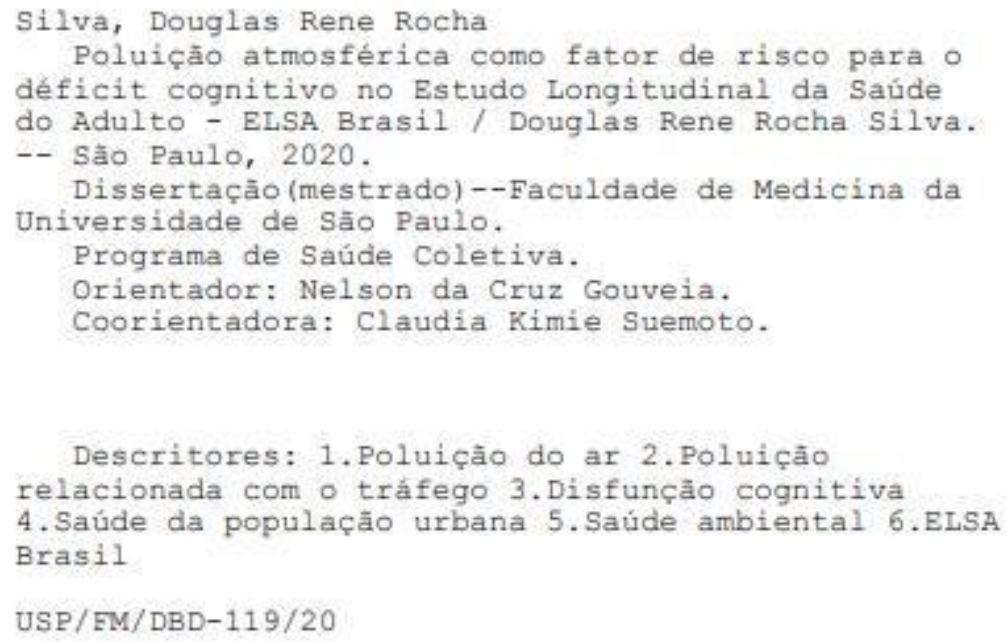

Responsável: Erinalva da Conceiçăo Batista, CRB-8 6755 


\section{DEDICATÓRIA}

À minha família 


\section{AGRADECIMENTOS}

Ao meu orientador, Nelson Gouveia, pela confiança, dedicação e incentivo.

À minha co-orientadora, Claudia K. Suemoto, pela confiança, empenho e dedicação.

Aos professores do programa de Saúde Coletiva da FMUSP pelas experiências que me proporcionaram ao longo de minha formação.

Aos calaboradores dos manuscritos que compuseram esta dissertação.

E por fim, um agradecimento mais que especial aos colegas Lucas Soriano, Claudio Kanai, Felipe Nascimento e Míriam Regina, pelo suporte tecnológico e estatistico ao longo deste projeto, além dos momentos de descontração. 
Stop.

A vida parou

ou foi o automóvel?

Carlos Drummond de Andrade 
Esta dissertação está de acordo com as seguintes normas, em vigor no momento desta publicação:

Referências: adaptado de International Committee of Medical Journals Editors (Vancouver). Universidade de São Paulo. Faculdade de Medicina. Divisão de Biblioteca e Documentação. Guia de apresentação de dissertações, teses e monografias. Elaborado por Anneliese Carneiro da Cunha, Maria Julia de A. L. Freddi, Maria F. Crestana, Marinalva de Souza Aragão, Suely Campos Cardoso, Valério Vilhena. $3^{\mathrm{a}}$ ed. São Paulo: Divisão de Biblioteca e Documantação; 2011.

Abreviaturas dos títulos dos periódicos de acordo com List of Journals Indexed in Index Medicus. 


\section{LISTA DE ABREVIATURAS E SIGLAS}

OMS Organização Mundial de Saúde

RR Risco Relativo

COMEAP Comitê Médico de Poluição Atmosférica

MP10 Material Particulado de 10 micrômetros

CO Monóxido de Carbono

HC Hidrocarbonetos

NOx $_{\mathbf{x}}$ Óxido de Nitrogênio

SOx Óxido de Enxofre

SNC Sistema Nervoso Central

BHE Barreira Hematoencefálica

TLR Receptores do tipo Toll

LPS Lipopolissacarídeo

$\mathbf{M P}_{2,5}$ Material Particulado de 2,5 micrômetros

$\mathrm{NO}_{2}$ Dióxido de Nitrogênio

$\mathrm{O}_{3}$ Ozônio

ELSA- Estudo Longitudinal de Saúde do Adulto

BRASIL

FIOCRUZ Fundação Oswaldo Cruz

UFBA Universidade Federal da Bahia

UFES Universidade Federal do Espirito Santo

UFRGS Uiversidade Federal do Rio Grande do Sul

USP Universidade de São Paulo

CERAD Consortium to Establish a Registry for Alzheimer's Disease

BBRC Bateria Breve de Rastreio Cognitivo

DTPD Densidade de Trafego Ponderada pela Distância

CET Companhia de Engenharia de Tráfego

DTPD_Comb Densidade de Trafego Ponderada pela Distância Combinada

DTPD_Trab Densidade de Trafego Ponderada pela Distância Trabalho

DTPD_Casa Densidade de Trafego Ponderada pela Distância Casa

DN Doenças Neurológicas 


\section{LISTA DE QUADROS E FIGURAS}

Figura 1: Efeitos globais da exposição aos poluentes ambientais na saúde humana.................................. 17

Figura 2: Mecanismos fisiopatológicos dos poluentes atmosféricos nos diversos órgãos e sistemas. 19

Figura 3: Projeção do número de pessoas com demência até 2050 em países pobres e em desenvolvimento em comparação com países desenvolvidos.

Figura 4: O impacto da poluição atmosférica no sistema nervoso central através de múltiplos caminhos 23

Figura 5: Centros de investigação que compõem o ELSA-Brasil .26

Figura 6: A) Representação esquemática das palavras apresentadas ao voluntário no teste de palavras

CERAD. B) Representação esquemática do voluntário recordando as palavras apresentadas.

Figura 7: Representação esquemática do teste de evocação de palavras

Figura 8: A) Representação esquemática do teste de reconhecimento de palavras. B) Representação esquemática do voluntário reconhecendo apenas as palavras apresentadas e não as confundidoras.

Figura 9: Representação esquemática dos testes de fluência verbal fonêmico (letra "F") e semântico (animais) 31

Figura 10: Representação esquemática do Teste de Trilha versão B 32

Figura 11: Representação de um buffer com 750 pés $(228,4 \mathrm{~m})$ de raio no entorno da residência de um sujeito, em um estudo epidemiológico 


\section{LISTA DE TABELAS}

Tabela 1: Descrição dos testes de cognição utilizados no estudo ELSA-Brasil................................. 28 


\section{SUMÁRIO}

LISTA DE ABREVIATURAS E SIGLAS .................................................................. 8

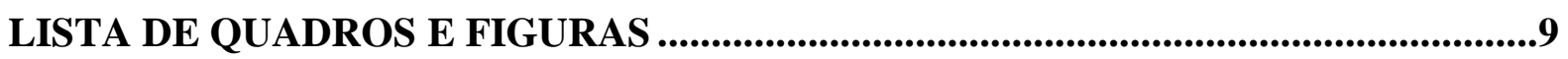

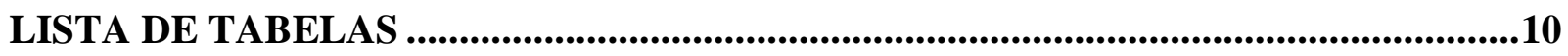

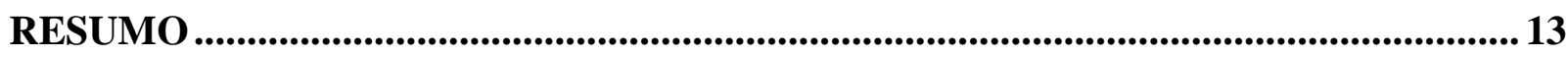

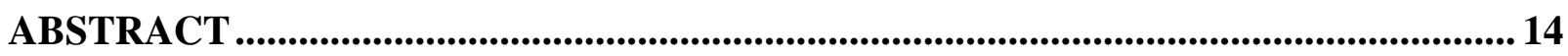

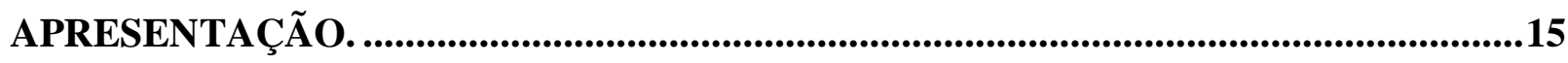

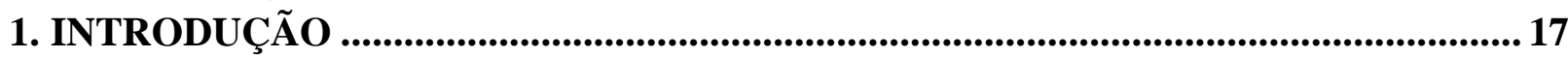

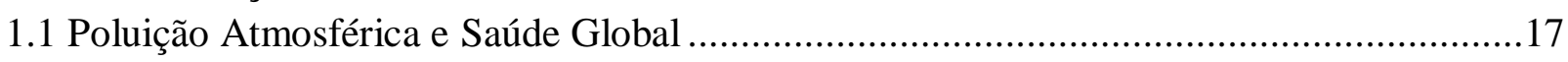

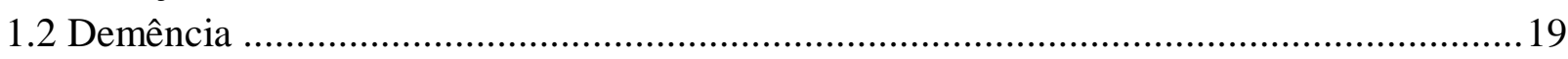

1.3 Fisiopatologia do Comprometimento Cognitivo e Demência Associados à

Exposição aos Poluentes Atmosféricos ...................................................................... 21

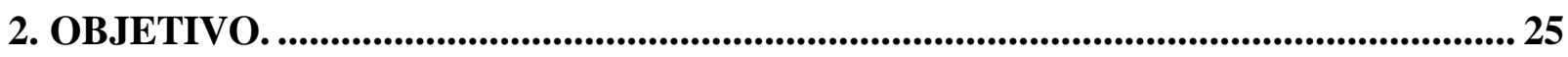

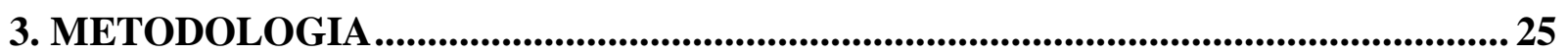

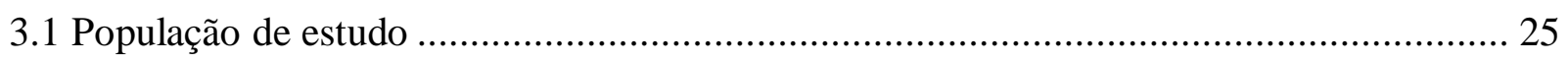

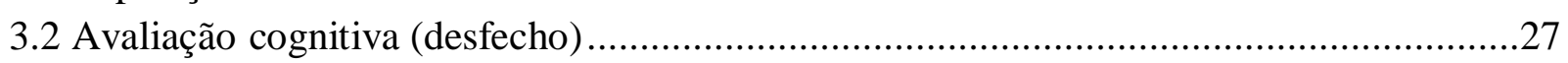

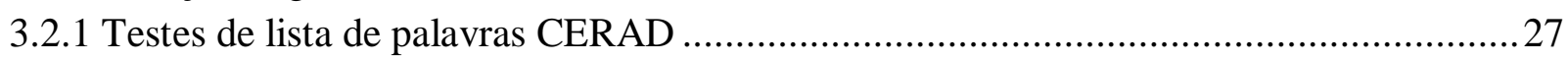

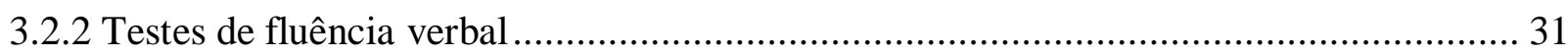

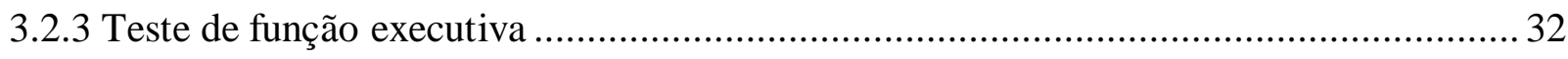

3.3 Avaliação dos poluentes atmosféricos (variável de exposição) ....................................... 33

3.3.1 Obtenção dos dados viários e de tráfego................................................................... 33

3.3.2 Cálculo da densidade de tráfego ponderada pela distância (DTPD) ............................... 34

3.3.3 Cálculo da densidade de tráfego ponderada pela distância combinada entre

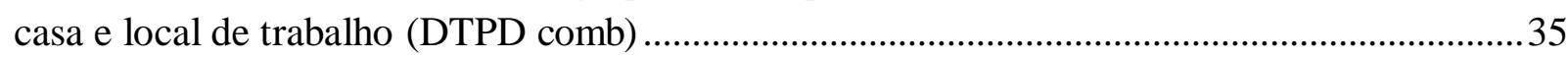

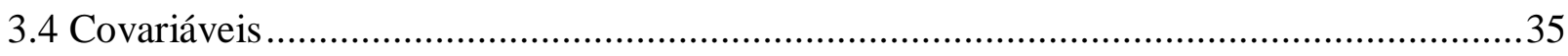

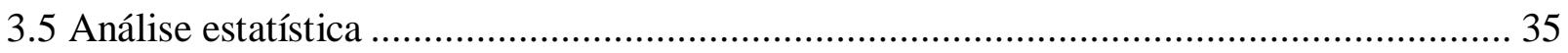

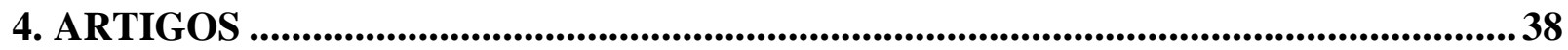

\section{AIR POLLUTANTS AS A RISK FACTOR FOR COGNITIVE IMPAIRMENT AND DEMENTIA}

5.1 Introduction

5.1.1 Population aging and cognition

5.1.2 Air pollutants and human health

5.1.3 Timeliness of the study

5.1.4 Final remarks

VEHICULAR TRAFFIC DENSITY AND COGNITIVE PERFORMANCE IN THE ELSABRASIL STUDY

Abstract

6.1 Introduction

6.2 Material and Methods

6.3 Results 
6.4 Discussion

6.5 Conclusion

6.6 References

6.7 Supplementary Material

5. CONSIDERAÇÕES FINAIS ..............................................................................................64

6. REFERÊNCIAS BIBLIOGRÁFICAS...........................................................................67

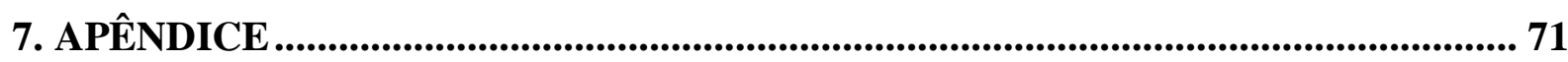
APÊNDICE A: PARECER CEP DA PESQUISA-BASE 


\section{RESUMO}

Silva DRR. Poluição atmosférica como fator de risco para o déficit cognitivo no Estudo Longitudinal da Saúde do Adulto - ELSA Brasil. São Paulo: Faculdade de Medicina, Universidade de São Paulo; 2020.

Introdução: Apesar do conhecimento sobre os efeitos deletérios dos poluentes atmosféricos na mortalidade e morbidade por problemas respiratórios e cardiovasculares, pouco se sabe sobre a relação entre esses poluentes e as doenças neurológicas. Recentemente, alguns estudos têm demonstrado uma possível associação entre exposição a poluentes atmosféricos de origem urbana e comprometimento cognitivo. Partindo desse contexto, nossa hipótese é investigar se existe associação entre a exposição aos poluentes atmosféricos e o desempenho cognitivo em pessoas expostas à poluição do ar na cidade de São Paulo. Objetivos: Investigamos os possíveis efeitos da exposição à poluição atmosférica sobre o desempenho cognitivo em participantes do Estudo Longitudinal da Saúde do Adulto (ELSA-Brasil) na cidade de São Paulo. Metodologia: Analisamos informações sobre função cognitiva, por meio dos escores de três testes validados e padronizados para a população brasileira: o teste de palavras CERAD; o teste de fluência verbal; e o teste de trilhas. A avaliação da exposição aos poluentes atmosféricos foi feita indiretamente, utilizando-se a densidade de tráfego ponderada pela distância (DTPD) na residência, no trabalho e a combinada (residência/trabalho). Na análise, usamos modelos de regressão linear simples e ajustados para idade, sexo, etnia, escolaridade, tabagismo, depressão, uso de álcool, diabetes, LDL-colesterol, HDL-colesterol, doenças cardiovasculares, hipertensão arterial sistêmica e atividade física. Resultados: Entre os 5.061 participantes do ELSA-Brasil- São Paulo, foram incluídos 4.415 (média de idade $=51,4 \pm 9,6$ anos, $46 \%$ homens e $60 \%$ brancos). Nos modelos de regressão linear simples, os participantes do terceiro tercil de DTPD combinado (residência e trabalho) apresentaram melhor desempenho global, em memória e fluência verbal quando comparados aos participantes do primeiro tercil. Por outro lado, os participantes do terceiro tercil de DTPD apresentaram pior desempenho cognitivo no teste de trilha (TMT) quando comparados aos participantes do primeiro tercil ( $\mathrm{p}<0,0001)$. No entanto, a DTPD não foi associada ao desempenho cognitivo em modelos ajustados. Quando investigamos a associação do desempenho cognitivo com a DTPD perto do trabalho e da residência dos participantes separadamente, encontramos resultados semelhantes.

Descritores: Poluição do ar; Poluição relacionada com o tráfego; Disfunção cognitiva; Saúde da população urbana; Saúde ambiental; ELSA Brasil. 


\begin{abstract}
Silva DRR. Air pollution as a risk factor for cognitive impairment in the Longitudinal Study of Adult Health - ELSA Brasil. São Paulo: "Faculdade de Medicina, Universidade de São Paulo; 2020.

Introduction: Despite knowledge about the deleterious effects of air pollutants on mortality and morbidity due to respiratory and cardiovascular problems, little is known about the relationship between these pollutants and neurological diseases. Recently, some studies have demonstrated a possible association between exposure to air pollutants of urban origin and cognitive impairment. Based on this context, our hypothesis is to investigate whether there is an association between exposure to air pollutants and cognitive performance in people exposed to air pollution in the city of São Paulo. Objectives: We investigated the possible effects of exposure to air pollution on cognitive performance in participants of the Longitudinal Study of Adult Health (ELSA-Brasil) in the city of São Paulo. Methodology: We analyzed information on cognitive function, through the scores of three validated and standardized tests for the Brazilian population: the CERAD word test; the verbal fluency test; and testing the trails. The assessment of exposure to air pollutants was made indirectly, using the distance-weighted traffic density (DTPD), at home, at work and combined (residence / work). In the analysis, we used simple linear regression models adjusted for age, sex, ethnicity, education, smoking, depression, alcohol use, diabetes, LDL-cholesterol, HDL-cholesterol, cardiovascular diseases, systemic arterial hypertension and physical activity. Results: Among the 5,061 participants at ELSA-Brasil-São Paulo, 4,415 were included (mean age $=51.4 \pm 9.6$ years, $46 \%$ men and $60 \%$ white). In the simple linear regression models, participants in the third tertile of combined DTPD (residence and work) showed better overall performance, in memory and verbal fluency when compared to participants in the first tertile. On the other hand, participants in the third tertile of DTPD showed worse cognitive performance in the trail test (TMT) when compared to participants in the first tertile $(\mathrm{p}<0.0001)$. However, DTPD was not associated with cognitive performance in adjusted models. When we investigated the association of cognitive performance with DTPD near work and the participants' residence separately, we found similar results.
\end{abstract}

Descriptors: Air pollution; Traffic-related pollution; Cognitive dysfunction; Urban health; Environmental health; ELSA Brasil. 


\section{APRESENTAÇÃO}

A preocupação com os efeitos da poluição atmosférica na saúde humana tem início em meados do século XVIII, contudo esses efeitos só foram amplamente descritos a partir da segunda metade do século XX (OLIVEIRA, 2006). O primeiro registro ocorreu no Vale Meuse - Bélgica, uma região que concentrava muitas indústrias em 1930 e no inverno daquele ano ocorreram 60 mortes por doenças respiratórias, número que foi considerado muito alto quando comparado aos dados dos anos anteriores e, assim, foi atribuido à exposição aos poluentes atmosféricos (FIRKET, 1931). Em 1948, na cidade de Donora, Pensilvânia - EUA, uma inversão térmica foi associada a 20 mortes por problemas respiratórios, influenciados pela poluição gerada por indústrias locais (CIOCCO, 1961). No entanto, foi o inverno londrino de 1952 que, sob a ação de uma inversão térmica, agravado pela elevada emissão de poluentes atmosféricos oriundos na produção industrial, resultou em um aumento de 4 mil mortes em três dias em relação à média de óbitos em períodos semelhantes anteriores (LOGAN, 1952). Nos dias de hoje, os dados da Organização Mundial de Saúde (OMS) apontam que, sozinhos, os poluentes atmosféricos representam uma grande ameaça à saúde e ao clima, visto que ao combinarmos os efeitos deletérios das emissões atmosféricas de origem externa (indústrias, automóveis, etc) e interna (queima de combustível fóssil para preparação de alimentos), cerca de 7 milhões de óbitos prematuros por ano são atribuídos a essas emissões, sendo, em grande parte, resultado do aumento da mortalidade por acidentes vasculares cerebrais, doenças cardíacas, doença pulmonar obstrutiva crônica, câncer de pulmão e infecções respiratórias agudas (WHO, 2006).

Foi justamente esse panorama que norteou, em 2017, meu ingresso no mestrado junto ao Departamento de Medicina Preventiva da Faculdade de Medicina da Universidade de São Paulo, com um objetivo muito claro: compreender o potencial dos poluentes atmosféricos, principalmente de origem veicular e em regiões urbanas, em ocasionar danos cognitivos em indivíduos adultos. Pretendendo, com isso, contribuir com o conhecimento e o debate científico acerca da exposição e emissão de poluentes atmosféricos em regiões urbanas e muito povoadas. Assim, fornecer evidências que possam contribuir com a fomulação de estratégias para mitigar a 
emissão de poluentes do ar e, consequentemente, minimizar os efeitos dessa exposição sempre foram pilares importantes nesta pesquisa.

Logo, o primerio capítulo desta dissertação apresenta o referencial teórico sobre os efeitos negativos na saúde ocasionados pela inalação de poluentes atmosféricos ao redor do mundo. Ainda neste capítulo, levantamos uma possível associação entre a exposição aos poluentes atmosféricos e danos cognitivos ocasionados ao longo de muitos anos de exposição, que poderão ou não se refletir em pior desempenho cognitivo e, em casos mais graves, em demência. O capítulo seguinte apresenta uma abordagem sobre nossa metodologia.

As sessões seguintes apresentam nossa produção ao longo deste estudo, são elas:

1 - Air Pollutants As A Risk Factor For Cognitive Impairment And Dementia (Silva DRR et al., 2019).

2- Vehicular Traffic Density And Cognitive Performance In The Elsa-Brasil Study (Silva, DRR et al., 2020). submitted.

O primeiro artigo teve como objetivo incitar a discussão em contexto principalmente nacional, mas também contribuir para a discussão em contexto internacional, sobre a possível correlação entre a exposição aos poluentes atmosféricos e pior desempenho cognitivo. Já o segundo artigo transcreve todo o esforço que tivemos ao longo desta pesquisa para evidenciar a possível associação entre poluição atmosférica e desempenho cognitivo no ELSA-Brasil.

Por fim, o capítulo de considerações finais aborda justamente as dificuldades que tivemos ao longo desta pesquisa, mas ressalta que existe sim uma associação entre os poluentes atmosféricos e pior desempenho cognitivo, mas que essa associação não pode ser estudada apenas sob a ótica da proximidade com o trafégo, mas também olhando para diversas condições socioecônomicas e clínicas. 


\section{INTRODUÇÃO}

\subsection{Poluentes Atmosféricos e Saúde Global}

Diversos poluentes, ao serem lançados diariamente de forma não controlada no ambiente, constituem uma grave ameça global à saúde dos seres vivos e foram responsáveis por cerca de 9 milhões de óbitos no mundo em 2015, o que corresponde a 16\% de todas as mortes ocorridas no mesmo ano (Figura 1) (McMichael 2017; Rockstrôm et al., 2009; Whitmee et al, 2015; Landrigan et al., 2017). Nesse contexto, quando olhamos especialmente para os poluentes emitidos por fontes industriais e escapamentos veiculares, verifica-se que a emissão de poluentes por essas fontes aumentou de forma exacerbada nos últimos 100 anos, colocando a poluição ambiental como a maior causa ambiental de doenças não transmissíveis e mortes prematuras do mundo (WHO, 2013).

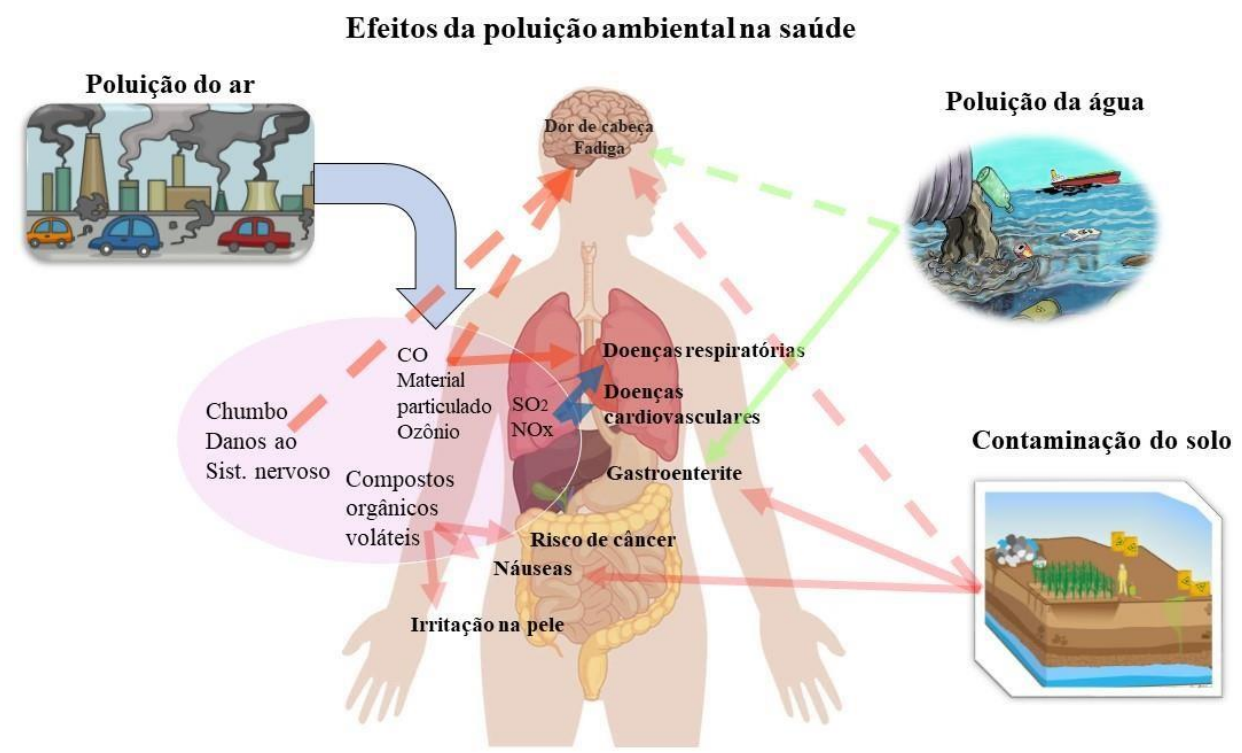

Figura 1: Efeitos globais da exposição aos poluentes ambientais na saúde humana.Adaptado: McMichael et al., 2017.

Nesse sentido, dados da Organização Mundial de Saúde (OMS) apontam que, sozinhos, os poluentes atmosféricos representam uma grande ameaça à saúde e ao clima, visto que, ao combinarmos os efeitos deletérios das emissões atmosféricas de origem 
externa (indústrias, automóveis, etc) e interna (queima de combustível fóssil para preparação de alimentos), cerca de 7 milhões de óbitos prematuros por ano são atribuídos a essas emissões, sendo, em grande parte, resultado do aumento da mortalidade por acidentes vasculares cerebrais, doenças cardíacas, doença pulmonar obstrutiva crônica, câncer de pulmão e infecções respiratórias agudas (WHO, 2006). Para a OMS, poluição atmosférica é toda e qualquer forma de matéria ou energia com intensidade e em quantidade suficiente para tornar o ar impróprio, nocivo ou ofensivo à saúde dos seres vivos (WHO, 2006). Além do mais, hoje estima-se que mais de $80 \%$ das pessoas que vivem em áreas urbanas que têm monitoramento de suas emissões de poluentes atmosféricos regularmente estão expostas a níveis de qualidade do ar que excedem os níveis preconizados pela OMS (WHO, 2019). Como consequência, estimativas para 2050 colocam os poluentes atmosféricos de origem urbana como o principal fator de morte por causas ambientais, superando as estimativas de morte por malária, consumo de água insalubre e falta de saneamento básico (OECD, 2011; Vormittag et al., 2013), o que reforça a necessidade de controlar essas emissões atmosféricas, para garantir a qualidade de vida nas grandes cidades.

No Brasil, apesar das limitações dos dados ambientais e de estudos que incorporem a variação do clima e da exposição em diferentes regiões do país, estima-se que o número de óbitos decorrentes da exposição aos poluentes atmosféricos seja algo próximo a 13 mil óbitos por ano associados ao material particulado (Pruss-Ustun, Bonjour, Corvalan, 2008). Porém, dados mais recentes e apenas para o Estado de São Paulo trazem projeções para os próximos 15 anos indicando que, se nada for feito, as mortes ocasionadas pelos poluentes atmosféricos irão somar 250 mil óbitos, um milhão de internações e vão custar aos cofres públicos 1,6 milhão de reais (Vormittag et al.,2013). Cabe ressaltar ainda que, atualmente, nos grandes centros urbanos, 
aproximadamente $90 \%$ dos poluentes lançados na atmosfera são emitidos pelo tráfego veicular (CETESB, 2012). Dentre os principais poluentes emitidos diariamente, que, em geral, são produtos da queima de combustíveis fósseis (IPEA, 2011), estão o monóxido de carbono $(\mathrm{CO})$, os hidrocarbonetos $(\mathrm{HC})$, os materiais particulados (MP), os óxidos de nitrogênio (NOx) e os óxidos de enxofre (SOx).

Essa emissão se torna relevante visto que, mesmo em baixas concentrações, os poluentes atmosféricos estão associados à morbidade e mortalidade por doença cardiopulmonar, exacerbação dos sintomas de asma e redução da função pulmonar, além de estarem associados a doenças neurológicas e possivelmente a comprometimento das funções cognitivas (Daniels et al., 2000; Li et al., 2003; Ostro et al., 2004; WHO, 2006; Kunzliet al., 2010) (Figura 2).

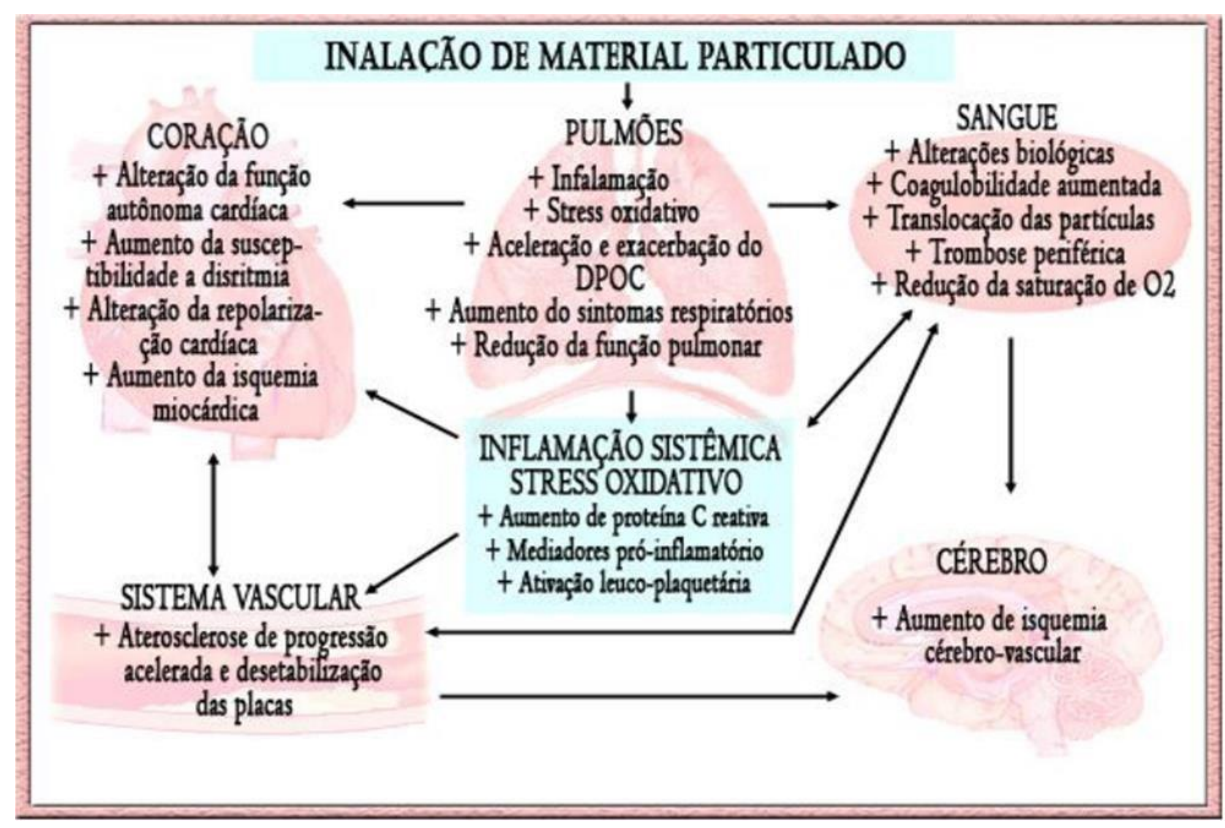

Figura 2: Mecanismos fisiopatológicos dos poluentes atmosféricos nos diversos órgãos e sistemas. Adaptado de Pope III (2006, p. 709-742, apud OLMO, 2010, p. 13). 


\subsection{Demência}

Atualmente, começa a ser estudada a relação entre a exposição aos poluentes atmosféricos e o desenvolvimento de doenças neurológicas, bem como o comprometimento cognitivo e demência. Embora o comprometimento cognitivo inicial, a princípio, não tenha impacto na vida social de seus portadores e não acarrete grandes restrições de funcionalidade na comunidade em que se inserem, casos mais graves em que a demência é manifestada levam à maior perda de funcionalidade e de qualidade de vida (Burlá et al., 2012; World Alzheimer's Disease International, 2009). Evidenciando, assim, a importância da atenção aos fatores de risco que levam ao comprometimento das funções cognitivas como alvo de políticas públicas de prevenção em um contexto global e emum futuro próximo. A OMS estimou que, em 2010, o número de pessoas com algum tipo de demência ao redor do mundo era de 35,6 milhões e que há projeção de que esse número irá dobrar a cada 20 anos, chegando a 65,7 milhões em 2030 e 115,4 milhões em 2050 (WHO, 2013). Dessa forma, o número total de novos casos de demência a cada ano ao redor do mundo é de aproximadamente 7,7 milhões, o que implica que um novo caso é diagnosticado a cada quatro segundos (WHO, 2013). Configurando-se, assim, como uma tendência mundial, que afeta particularmente países pobres e em desenvolvimento, como é o caso dos países que compõem os BRICS (Brasil, Rússia, Índia, China e África do Sul), tendo em vista que entre estes cinco países, quatro encontram-se entre os nove com maior número de pessoas com algum tipo de demência no mundo (WHO, 2013) (Figura 3). 


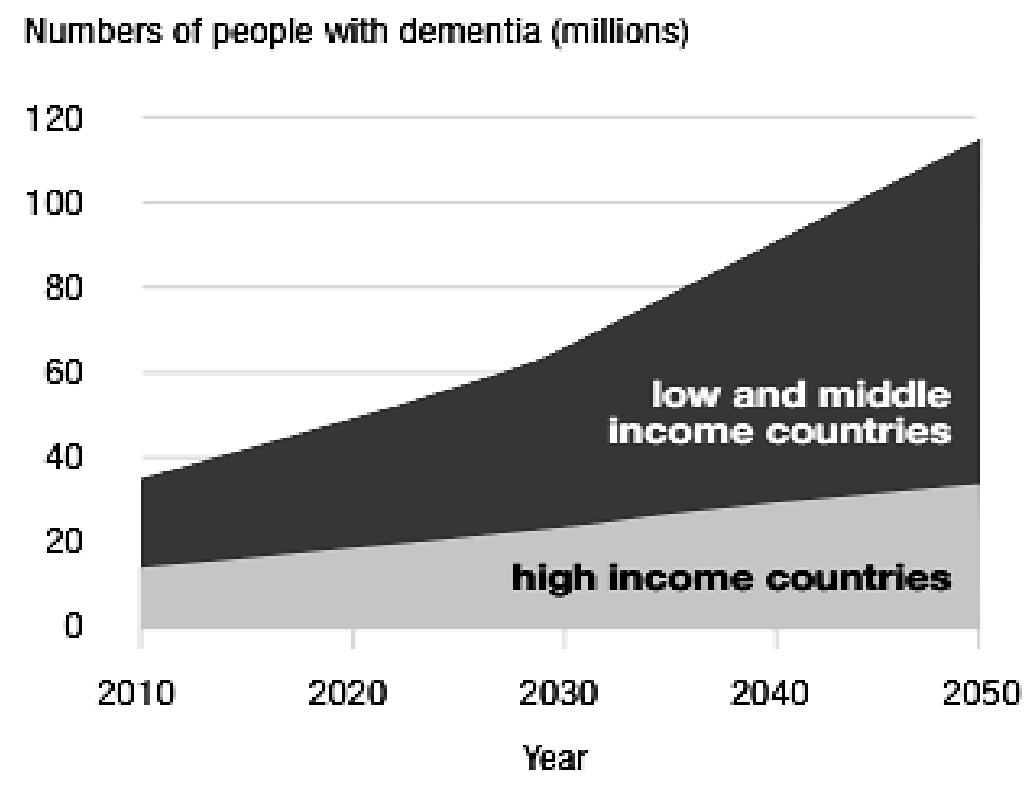

Figura 3: Projeção do número de pessoas com demência até 2050 em países pobres e em desenvolvimento em comparação com países desenvolvidos. Fonte: Relatório Mundial de Alzheimer, 2010.

\subsection{Fisiopatologia do Comprometimento Cognitivo e Demência}

\section{Associados a Exposição aos Poluentes Atmosféricos}

Conforme descrito por Calderón-Garcidueña e colegas em 2002 (CalderónGarciudeñas et al., 2002), os poluentes atmosféricos provocam efeitos deletérios no sistema nervoso central humano (SNC). Os poluentes atingem o SNC, principalmente, por quatro vias (Calderón-Garcidueñas et al., 2008), descritas a seguir:

$1^{\circ}$. A primeira delas é a via do bulbo olfatório, em que as partículas poluentes entram pelos forâmes da placa cribiforme na base do crânio e atingem o bulbo olfatório, invadindo o SNC e causando toxicidade neuronal.

$2^{\circ}$. Outra via é a sistêmica, sendo que nela as partículas e os poluentes aspirados entram na circulação sistêmica através dos alvéolos pulmonares. Uma vez no sangue, causam inflamação sistêmica com aumento de citocinas inflamatórias, o que leva à diminuição da permeabilidade vascular no cérebro e disfunção da barreira hematoencefálica (BHE), que, em condições 
homeostáticas, impede a entrada de poluentes no SNC (Tuppo et al.,2005).

$3^{\circ}$. Uma terceira via de acesso dos poluentes ao SNC é por transporte axonal. Nesse caso, substâncias exógenas se difundem para axônios de neurônios mais frequentemente expostos à poluição, como o trigêmio, pela proximidade com a cavidade nasal, e o vago, pela relação anatômica que guarda com os pulmões. Entrando pelos próprios neurônios, esses poluentes causam acúmulo de proteínas anômalas, como é o caso da proteína $\beta$ amilóide relacionada com a doença de Alzheimer, causa também disfunção de proteínas, como as chaperonas, que são responsáveis por corrigir erros na transmissão de informações genéticas e informações de organelas, podendo, assim, levar a um estado de estresse oxidativo, o que induz à morte celular programada, a apoptose (Nunomura et al.,2006). Se o processo é intenso, a morte celular é mais rápida, podendo causar necrose nas células nervosas.

$4^{\circ}$. Por fim, os poluentes atmosféricos impactam o SNC via inflamações produzidas pela ligação de poluentes a receptores de membrana das células da glia, os chamados Toll-like receptors (TLR), ligadores de lipopolissacarídeo (LPS), que desencadeiam uma sinalização intracelular produtora de citocinas e quimiocinas pró-inflamatórias, que culmina com a morte de neurônios (Genc et al.,2012). Essa via está mais associada a neurônios dopaminérgicos, daí sua estreita relação com a doença de Parkinson (Numan et al.,2015). 
Todas essas vias e seus efeitos estão esquematizados na Figura 4.

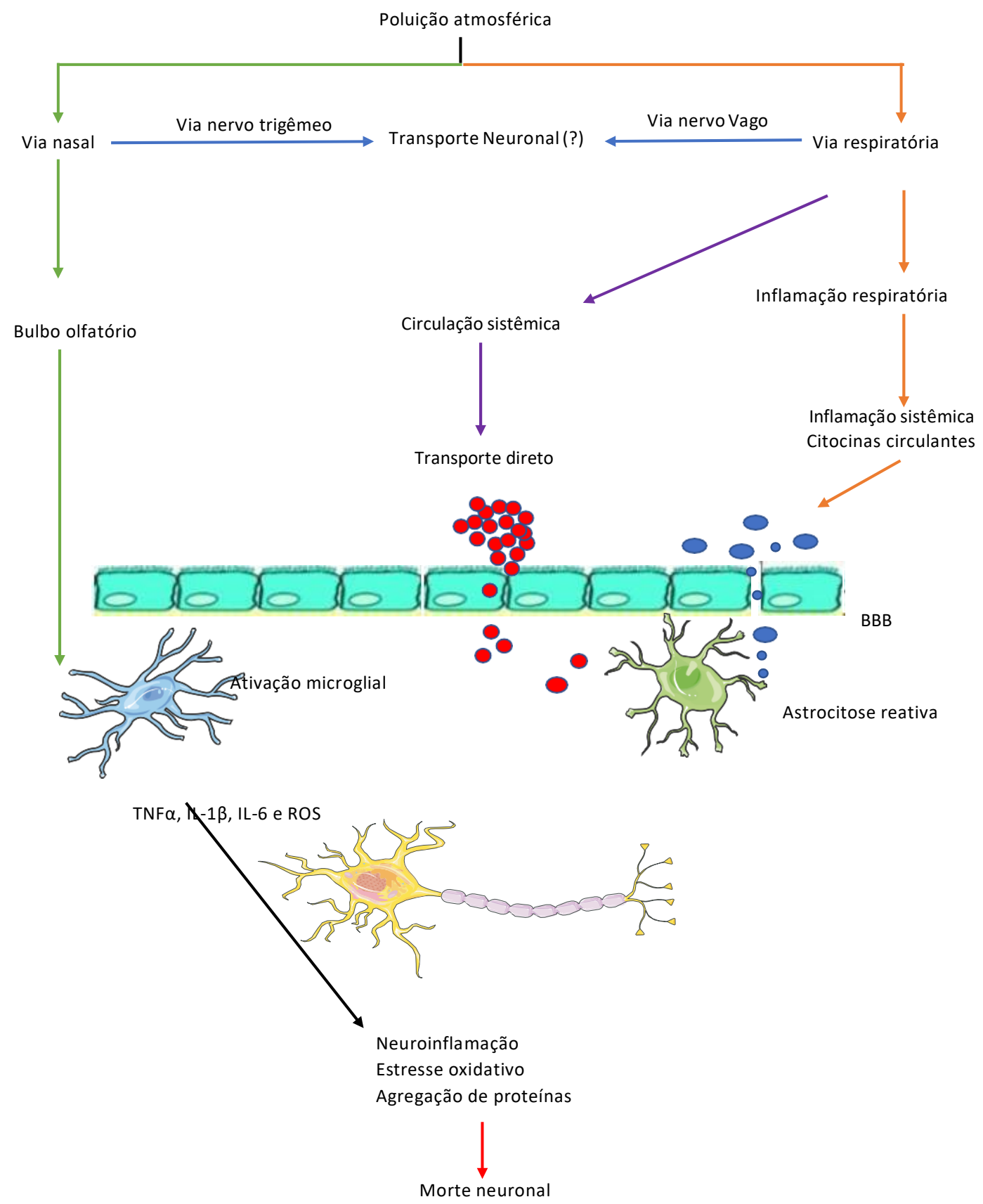

Figura 4: O impacto da poluição atmosférica no sistema nervoso central através de múltiplos caminhos. Adaptado de Genc et al., 2011 p3.

Estudos observacionais tambem têm demonstrado que os poluentes atmosféricos podem contribuir para o processo neurodegenerativo, inclusive desde a infância, especialmente em indivíduos expostos cronicamente a essa emissão (Blok et al., 2009; Calderon-Garcidueñas et al., 2004; Calderon-Garcidueñas et al., 2008; CalderonGarcidueñas et al., 2008). O estudo realizado por Chen et al., (2017), contendo 2,1 
milhões de pessoas, encontrou associação positiva entre o material particulado fino $\left(\mathrm{MP}_{2,5}\right)$, mesmo em baixas concentrações, e a incidência de demência na cidade de Ontário, Canadá (Chen et al., 2017). Outro estudo epidemiológico, realizado na Suécia com 1806 voluntários adultos, encontrou associação positiva entre os poluentes atmosféricos e o aumento na incidência de demência e doença de Alzheimer (Oudin et al., 2016). Em Taiwan, dois estudos de coorte, sendo um com 96.000 voluntários e outro com 30.000 voluntários, identificaram que a exposição aos poluentes $\mathrm{NO}_{2}, \mathrm{MP}_{2,5}, \mathrm{O}_{3}$ e $\mathrm{CO}$ também estava associada ao aumento da incidência de demência (Chang et al., 2014; Jung et al., 2015). Nos Estados Unidos, uma coorte de mulheres idosas expostas ao $\mathrm{MP}_{2,5}$ associou essa exposição ao aumento de Alzheimer (Cacciottolo et al., 2017). Além desses achados, evidências recentes têm sugerido que viver perto de estradas intensifica a exposição aos poluentes atmosféricos, que, por sua vez, foram associados ao aumento de comprometimento cognitivo em voluntários idosos nos Estados Unidos (Wellenius et al., 2012). Esse resultado é sustentado por estudos epidemiológicos realizados na Alemanha (Ranft et al., 2009) e pela famosa coorte de Framingham realizada nos Estados Unidos (Wilker et al., 2015).

Apesar do conhecimento clínico e epidemiológico disponível sobre a associação entre doenças neurológicas e poluentes atmosféricos, países emergentes carecem de estudos e dados conclusivos (Nitrini et al.,2009; Prince et al.,2013). Nesse contexto, a América Latina, e consequentemente o Brasil, não assume posição melhor, visto que, em uma revisão de literatura publicada por Nitrini e colaboradores em 2009, com o objetivo de identificar a prevalência e incidência de doenças neurológicas associadas aos poluentes atmosféricos por países da América Latina, foram encontrados apenas oito trabalhos publicados. Essa escassez de estudos pode estar associada à ausência de dados, ou à baixa disponibilidade dos mesmos, ou à disponibilidade de dados imprecisos (Fagundes et 
al.,2011). Uma outra problemática relacionada à associação entre a exposição aos poluentes atmosféricos e as doenças neurológicas, bem como comprometimento das funções cognitivas e demência, está relacionada a população alvo desses estudos, que em sua maioria são focados apenas na população idosa. É importante ressaltar que as alterações neuropatológicas associadas às demências podem ter inicío durante a idade adulta, mesmo antes dos 50 anos, e progredir de forma assintomática por 20 a 30 anos (Tuokko et al., 2003), mesmo que nem todos os componentes da função cognitiva sejam igualmente afetados.

Nesse sentido, a análise do banco de dados com testes neuropsicológicos gerado pelo Estudo Longitudinal da Saúde do Adulto no Brasil (ELSA-Brasil) é uma grande oportunidade para o entendimento de como os poluentes atmosféricos podem ser considerados fatores de risco para o comprometimento da função cognitiva em indivíduos de 35 a 74 anos, expostos na região metropolitana de São Paulo.

\section{OBJETIVO}

Avaliar a associação entre a exposição a poluentes atmosféricos medida indiretamente através da Densidade de Trafego Ponderada pela Distância e a performance cognitiva em participantes do estudo ELSA-Brasil em moradores do município de São Paulo.

\section{METODOLOGIA}

\subsection{População de Estudo}

Para este estudo, utilizamos dados da primeira avaliação do ELSA-Brasil realizada entre 2008 e 2010. O ELSA-Brasil, com 15.105 participantes, é o maior estudo epidemiológico multicêntrico de coorte já desenvolvido na América Latina, com o 
objetivo principal de investigar doenças crônicas, como diabetes e doenças cardiovasculares, assim como os fatores de risco associados na população brasileira. Uma outra linha de investigação dentro do ELSA-Brasil busca compreender a história natural do envelhecimento cognitivo. O ELSA-Brasil é uma coorte composta por mulheres e homens na faixa etária dos 35 a 74 anos, servidores públicos de seis instuições de ensino e pesquisa das regiões Nordeste, Sudeste e Sul do Brasil, são elas: a Fundação Oswaldo Cruz (Fiocruz), a Universidade Federal da Bahia (UFBA), a Universidade Federal do Espírito Santo (UFES), a Universidade Federal de Minas Gerais (UFMG), a Universidade Federal do Rio Grande do Sul (UFRGS) e Universidade de São Paulo (USP) (Figura 5).

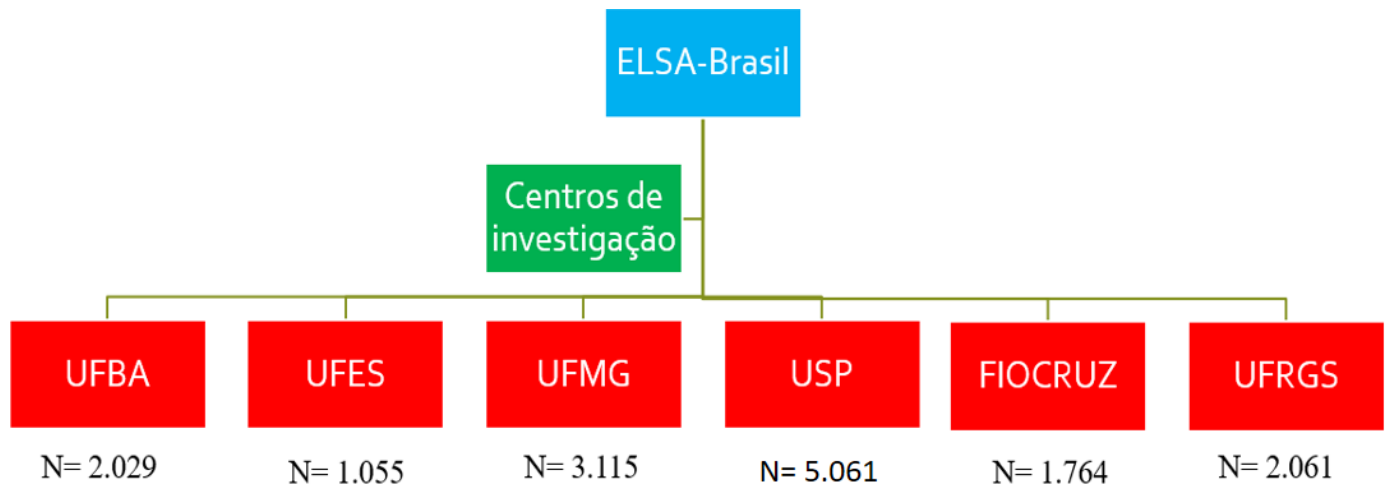

Figura5: Centros de investigação que compõem o ELSA-Brasil.

Como critério de exclusão para o ELSA-Brasil, foram definidos: a intenção de deixar a instituição, estar grávida ou ter estado a menos de quatro meses de puerpério, apresentar grave dificuldade cognitiva ou de comunicação e, se aposentado, residir fora da região metropolitana correspondente. Para melhor distribuição da população no estudo, foram definidos critérios e metas de recrutamento por sexo (50\% cada), idade ( $15 \%$ de 35-44 anos, 30\% 45-54 anos, $40 \%$ de 55-64 anos e 15\% de 65-74 anos) e categoria ocupacional (35\% do nível de apoio, com ensino fundamental incompleto; 35\% do nível médio e 30\% do nível superior/docente) (Aquino et al., 2012).

Em nosso estudo, utilizamos uma parcela do ELSA-Brasil composta por 5.061 
voluntários de ambos os sexos com idade entre 35 e 74 anos, servidores civis ativos e aposentados da Universidade de São Paulo. Do banco de dados da linha de base do estudo ELSA-Brasil foram obtidos dados georreferenciados a partir do endereço residencial e de trabalho, dados sobre condições sociais, história pregressa sobre a saúde com informações sobre doenças crônicas pré existentes, uso de medicamentos, hábitos alimentares e pessoais e dados sobre as condições atuais do ponto de vista físico e mental. Este estudo é restrito apenas aos participantes do centro de estudo de São Paulo, onde foi medida a densidade de tráfego ponderada de distância (DTDP). Para esta análise, foram excluídos os participantes com diagnóstico médico autorreferido de AVC no início do estudo, a fim de minimizar o efeito da doença cerebrovascular prévia na função cognitiva. Também foram excluídos os participantes que estavam usando medicações que indicam a presença de doenças neurológicas ou psiquiátricas ativas, ou que poderiam interferir diretamente no desempenho dos testes cognitivos (agentes antiparkinsonianos, antipsicóticos e anticonvulsivantes). Por fim, excluímos participantes com dados incompletos para os testes cognitivos ou medidas de DTPD. O estudo ELSA-Brasil foi aprovado pelos conselhos locais de revisão institucional e todos os participantes assinaram um termo de consentimento informado no momento da inscrição. Este estudo, em particular, também foi aprovado pelo Comitê de Ética em Pesquisa do Hospital Universitário da Universidade de São Paulo (CAAE: 96808518.9.0000.0076 - CEP-HU/USP: 1741/18).

\subsection{Avaliação Cognitiva (Desfecho)}

A escolha dos testes para avaliação cognitiva baseou-se nos seguintes critérios:

$1^{\circ}$. avaliação de múltiplos domínios cognitivos: memória (testes de aprendizagem, retenção e reconhecimento de palavras); linguagem [testes de fluência verbal semântica (animais) e fonêmica (primeira letra)]; e funções executivas (teste das trilhas versão $B$ ) (Tabela 1); 
$2^{\circ}$. comparabilidade nacional e internacional;

$3^{\circ}$. adaptação ao português;

$4^{\text {o }}$. regras normativas para diagnosticar déficits cognitivos;

$5^{\circ}$. compatibilidade entre o tempo de aplicação do teste cognitivo e o tempo reservado para todo o questionário ELSA-Brasil.

Visando à padronização da aplicação, foi elaborado o "Manual de Padronização para Aplicação de Testes Cognitivos" e oferecido aos aplicadores um treinamento para, assim, garantir e controlar a qualidade das informações colhidas para o estudo (Passos et al., 2014).

Tabela 1: Descrição dos testes cognitivos utilizados no estudo ELSA-Brasil (Estudo Longitudinal de Saúde do Adulto)

\begin{tabular}{|c|c|c|}
\hline Teste & Domínio principal & Capacidades de domínio \\
\hline $\begin{array}{l}\text { Palavras CERAD* } \\
\text { (Teste da figura }{ }^{\dagger} \text { ) } \\
\text { Evocação de palavras } \\
\text { (Figura de evocação) } \\
\text { Reconhecimento de palavras } \\
\text { (Reconhecimento de figura) } \\
\text { Testes de fluência verbal (animais e letra F) } \\
\text { Teste de trilha (versão B) }\end{array}$ & 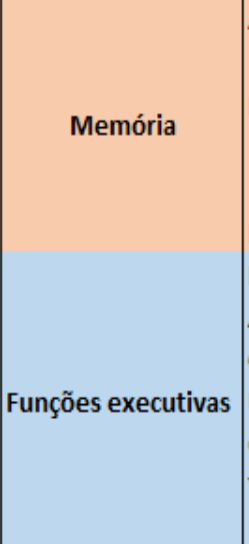 & $\begin{array}{l}\text { Aprendizagem imediata de novas informações; retenção de informações após três tentativas } \\
\text { Lembrando tardiamente informações aprendidas } \\
\text { Lembrando e distinguindo as palavras corretas entre um conjunto de palavras distratoras } \\
\text { Linguagem (também memória semântica) para os testes de fluência verbal } \\
\text { Atenção para uma tarefa ao longo do tempo } \\
\text { Concentração } \\
\text { Uso da informação aprendida nas instruções do teste } \\
\text { Organização visual-espacial; conectando palavras e números } \\
\text { Velocidade de processamento (falar palavras corretas ou conectar palavras e números) } \\
\text { Flexibilidade cognitiva: reconhecimento e correção de possíveis erros }\end{array}$ \\
\hline
\end{tabular}

* Palavras CERAD, versão brasileira: poste, motor, carta, braço, manteiga, erva, cabana, árvore, bilhete, praia (Bertolucci et al., 1998). †Figuras do teste de imagem: sapato, casa, pente, avião, chave, balde, tartaruga, livro, colher, árvore (Nitrini et al., 2004).

\subsubsection{Teste de lista de palavras do CERAD}

Os testes para avaliar memória têm por objetivo captar esta complexa função cognitiva, encarregada de reter informações ou percepções, para que possam ser posteriormente utilizadas. A recepção, o armazenamento e a liberação da informação são propriedades generalizadas da rede neurônica e relacionam-se à adaptação do indivíduo ao meio ambiente (Consenza et al., 2005).

O primeiro teste para avaliação da memória utilizado foi o teste de lista de 
palavras, adaptado para estudos brasileiros a partir do Consortium to Establish a Registryfor Alzheimer's disease (CERAD). Este teste inclui uma lista com dez palavras (poste, motor, carta, braço, manteiga, erva, cabana, árvore, bilhete, praia), não relacionadas, impressas em tamanho grande, para que seja mostrada aos voluntários em uma frequência entre 1 e 2 segundos cada (Figura 6A). Após o termino da apresentação das dez palavras, é solicitado aos voluntários que recordem o maior número de palavras que puderem em voz alta (Figura 6B). Os testes foram realizados três vezes, com pontuação máxima de 30 pontos e em cada uma das vezes a ordem das palavras foi diferente.

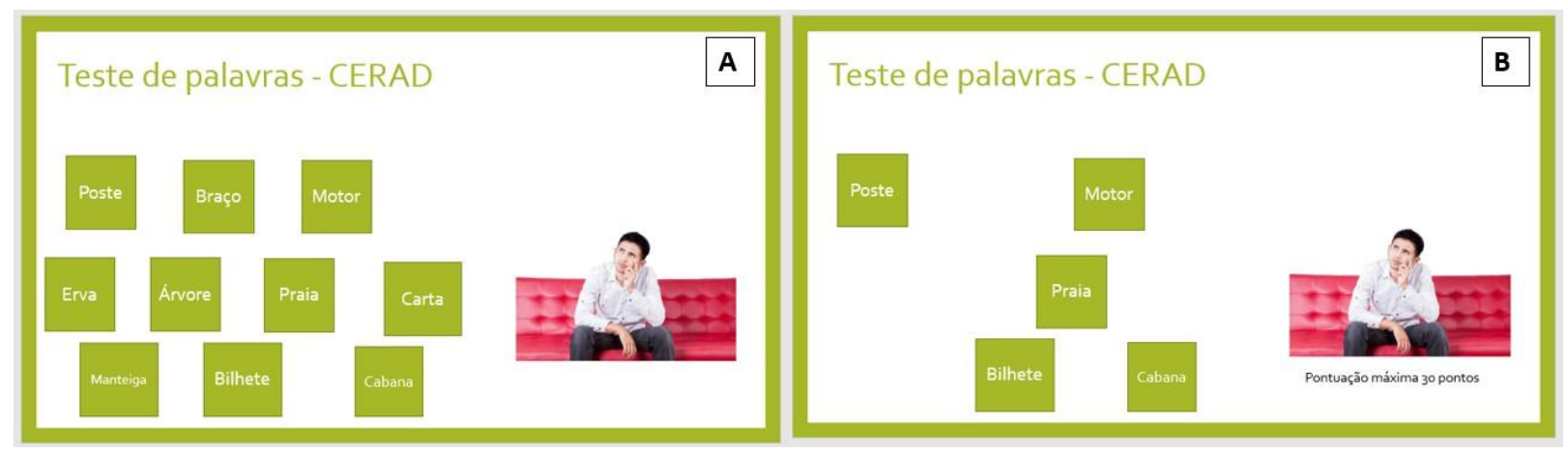

Figura 6: Representação esquematica das palavras apresentadas ao voluntário no teste de palavras CERAD (A), e voluntário recordando as palavras apresentadas (B).

Após aproximadamente cinco minutos de realização do teste de lista de palavras CERAD, e com a realização de uma atividade distratora, foi realizado o segundo teste para avaliação de memória, através do teste de evocação tardia. Este teste tem por objetivo avaliar a memória tardia e mede a capacidade de retenção das palavras ditas anteriormente. Assim, em cinco minutos o voluntário deveria recordar o maior número de palavras possíveis utilizadas no teste anterior (Figura 7) (Bertolucci et al., 1998). 


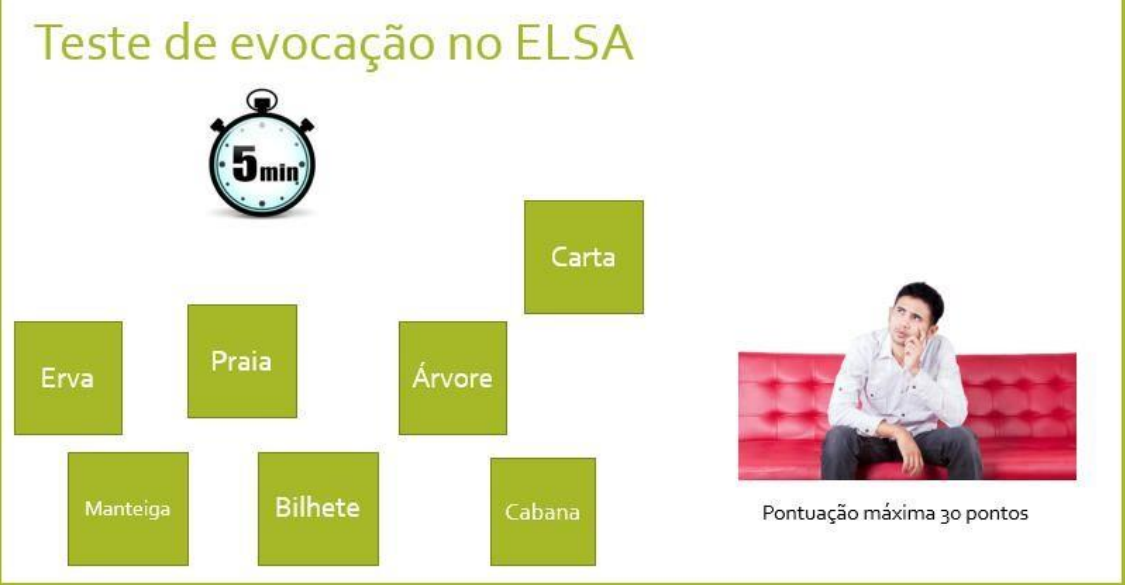

Figura 7: Representação esquemática das palavras apresentadas ao voluntário no teste de evocação de palavras.

O teste de reconhecimento de palavras foi o último teste utilizado para avaliar a memória (Figura 8). Este teste mede a capacidade de resgaste de informações com estímulos, por meio de uma lista de palavras que contém elementos da lista original, além de uma série de palavras distratoras (Nitrini et al., 2009).

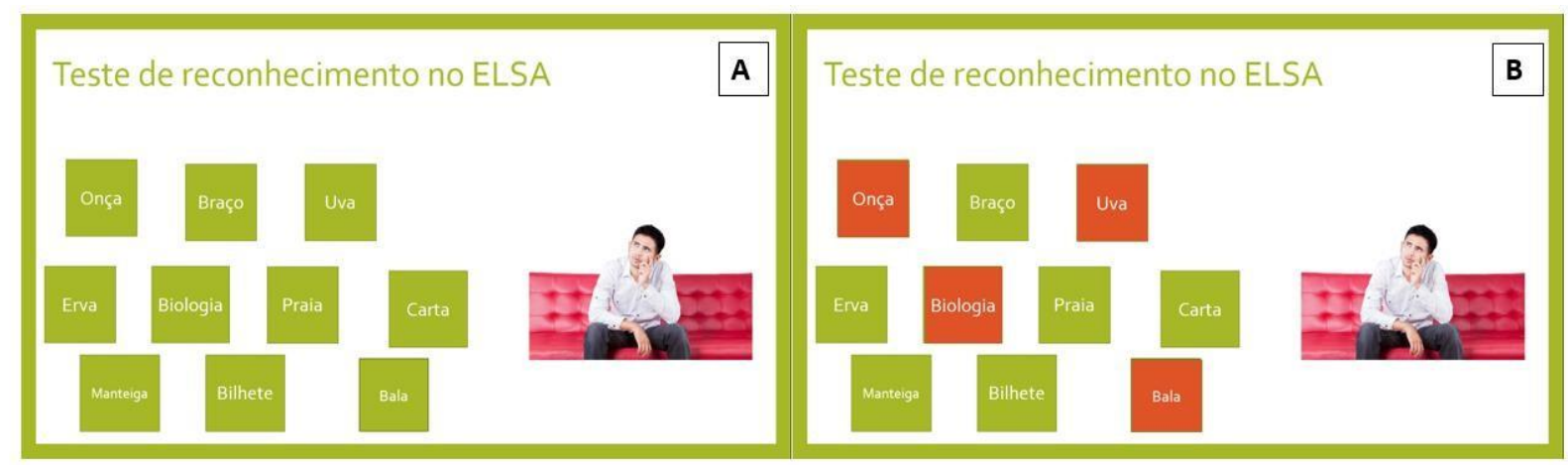

Figura 8: A) Representação esquemática das palavras apresentada ao voluntário no teste de reconhecimento de palavras.

B) Representação esquemática das palavras apresentada ao voluntário para reconehcimento apenas das palavras apresentadas e não as confundidoras.

Para os participantes analfabetos, foi utilizado o teste de figuras da bateria breve de rastreio cognitivo, contendo as imagens de (sapato, casa, pente, avião, chave, balde, tartaruga, livro, colher, árvore). Este teste traz a vantagem de seguir a mesma dinâmica que o teste de palavras (Nitrini et al., 2004). 


\subsubsection{Testes de Fluência Verbal}

Para avaliação da linguagem, foram utilizados os testes de fluência verbal semântica e de fluência verbal fonêmica. A fluência verbal é mensurada pela quantidade de palavras de uma mesma categoria lembradas em um determinado período de tempo, geralmente um minuto (Lezak et al., 1995). Os testes de fluência podem ser utilizados para avaliar a capacidade de armazenamento da memória verbal (léxico e semântico), a velocidade psicomotora, a linguagem (em que se pode avaliar a forma de expressão e como a pessoa utilizou o tempo disponível para execução do teste), a habilidade de recuperar a informação guardada na memória e as funções executivas, com foco na concentração, na capacidade de organizar o pensamento e nas estratégias utilizadas para a busca de palavras (Machado et al., 2005; Brucki et al., 1997; Lezak et al., 1995).

O teste de fluência verbal semântico consiste em pedir aos participantes para dizer o máximo de palavras possíveis em relação a uma categoria específica, que no caso é de animais, em um minuto. Já no teste de fluência verbal fonêmico, o voluntário deve dizer o máximo de palavras iniciadas com uma determinada letra também em um minuto. Na primeira coleta de dados do estudo ELSA-Brasil, foi utilizado a letra F (Figura 9). 


\section{Teste de Fluência Verbal no ELSA}

$>$ Fonêmica $(F, A, S)$

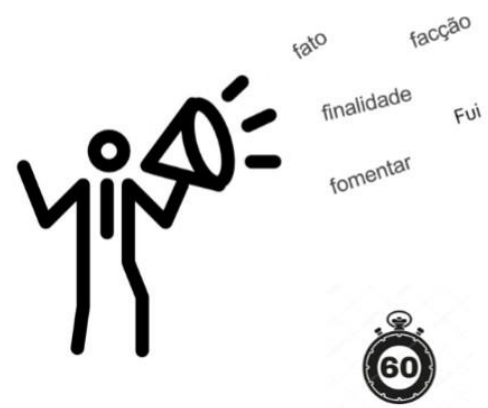

$>$ semântica (animais)

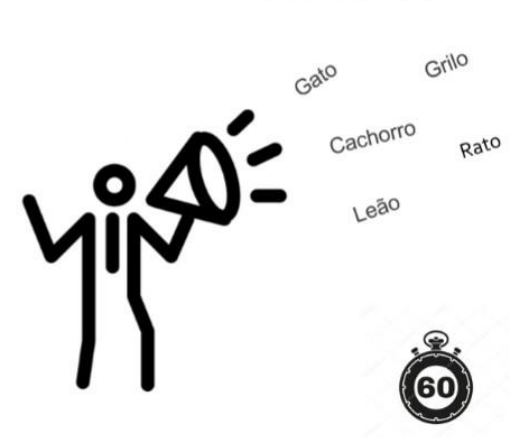

Figura 9: Representação esquemática dos testes de fluência verbal Fonêmico (Letra F) e Semêntico (Animais).

\subsubsection{Teste de função executiva}

O último teste utilizado para avaliação da função executiva é o teste de trilhas versão B (Trail-making), instrumento originalmente desenvolvido em 1944 para avaliação da rapidez visuomotora (Adam et al., 2005). É utilizado para examinar a velocidade de processamento, flexibilidade cognitiva, capacidade de manutenção do engajamento mental, o rastreamento visual, a destreza motora e a memória operacional (Consenza et al., 2005). Para realizar o teste de trilha versão B, o participante foi instruído a desenhar linhas, conectando letras e números em uma ordem que alternasse entre o aumento do valor numérico e a ordem alfabética (1, A, 2, B, 3, C etc.). O participante foi instruído a desenhar o mais rápido possível, sem levantar a ponta do lápis da página (Figura 10). Os supervisores foram instruídos a apontar os erros. O escore deste teste foi o tempo total gasto para completar a tarefa, incluindo o tempo necessário para corrigir erros (Bertolucci et al., 1998). 


\section{Teste de trilha versão B no ELSA}

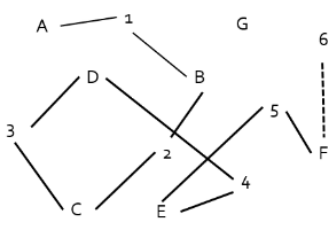

Figura 10: Representação esquemática do teste de Trilha Versão B.

\subsection{Avaliação da exposição aos poluentes atmosféricos}

\subsubsection{Obtenção dos dados viários e de tráfego}

Como variável de exposição, utilizamos a densidade de trafego ponderada pela distância (DTPD) relativa ao endereço residencial e de trabalho de cada voluntário do estudo. A DTPD foi calculada a partir das informações viárias e de tráfego veicular do ano de 2007 que correspondem ao período do início do estudo ELSA-Brasil. Estas informações incluem a base cartográfica das vias, por classificação, número de faixas de rolagem e velocidade máxima permitida no segmento, essa prática faz uso da medida mais direta dentre as disponíveis e foi aplicada em diversos estudos, tais como o de Wu et al. (2015), Oudin et al. (2016), Power et al. (2011), Chang et al. (2014), Tonne et al. (2014). Para este fim, a Companhia de Engenharia de Tráfego (CET), após contagem manual de veículos, utilizou o programa canadense EMME-2 para simular o tráfego nas vias coletoras I e II, arteriais I, II e III e trânsito rápido. Para os segmentos que não possuem valor de tráfego, foi atribuído o volume médio, de acordo com a classificação da respectiva via. O tráfego das vias locais foi estimado pela CET em 926 regiões delimitadas para planejamento de tráfego (denominadas Origem-Destino), sendo a soma de tráfego de cada uma dessas regiões dividida pela soma das extensões das vias locais 
contidas nelas e como resultado se obtém a densidade de tráfego (fluxo de veículos/metro de via local). Posteriormente, essa medida foi multiplicada pelo comprimento dos segmentos de vias locais ( $S V L)$, em metros, contidos nas respectivas regiões, obtendo-se o volume de tráfego de cada trecho de via local $(T)$.

$$
T=\left(\sum \text { fluxo: } \sum \text { comprimento }\right) \times S V L
$$

\subsubsection{Cálculo da densidade de tráfego ponderada pela distância (DTPD)}

Para cada sujeito do estudo já previamente georreferenciado e constante no banco de dados do estudo ELSA, foi calculada a DTPD em uma distância no entorno de sua residência e do local de trabalho (buffers), conforme a equação abaixo, na qual $L$ é largura do buffer e $D$ é a menor distância entre a casa e a via dentro do buffer, $Y$ é o valor utilizado para ponderar o fluxo veicular obtido para cada via dentro desta área.

$$
Y=\left(\frac{1}{0,4 \sqrt{2 \pi}}\right) \times \exp \left[\left(\frac{\left.(0,5)\left(\frac{D}{L}\right)^{2}\right)}{(0,4)^{2}}\right)\right]
$$

Divide-se, então, o fluxo veicular da referida via pelo valor de $Y$, gerando o valor de $X$. Então se somam os valores ponderados de tráfego $(X)$ de todas as vias contidas dentro do buffer (n), obtendo-se a DTPD individual de cada voluntário.

$$
X=\frac{\text { fluxo veicular }}{Y} \quad D T P D=\sum_{i=1}^{n} X_{i}
$$

Como a densidade e a característica das construções, direção predominante dos ventos, altitude e turbulência gerada pela passagem de veículos interferem na dispersão dos poluentes gerados pelo tráfego, pode-se assumir larguras variadas de buffers para calcular a DTPD, diminuindo as imprecisões na medida de exposição. Para estes cálculos foram utilizados buffers com raio de 150 metros, já previamentecalculados no âmbito de 
outro projeto de pesquisa (Figura 11).

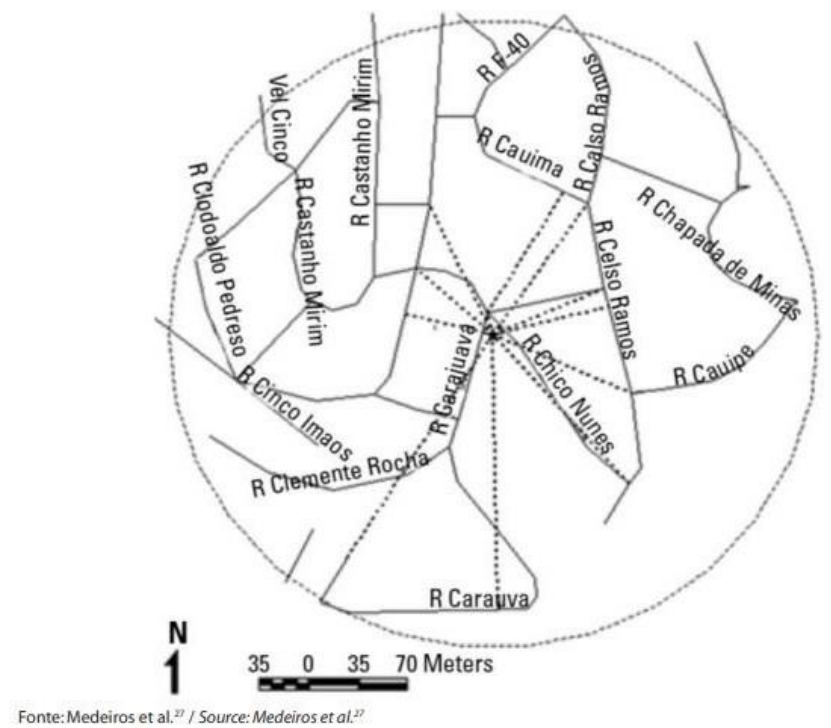

Figura 11: Representação de um buffer com 500 pés $(150 \mathrm{~m})$ de raio no entorno da residência de um sujeito, em um estudo epidemiológico.

\subsubsection{Cálculo da densidade de tráfego ponderada pela distância combinada}

\section{entre casa e local de trabalho (DTPD comb)}

Considerou-se horário comercial (8 horas) o tempo médio de permanência dos funcionários nas respectivas unidades da Universidade de São Paulo durante o período de trabalho. As demais horas do dia (16 horas) foram atribuídas a atividades fora da unidade ou à permanência em domicílio. Portanto, o cálculo da DPTD combinada (DTPD comb) foi realizado utilizando $1 / 3$ do valor da DTPD do local de trabalho (DTPD trab) mais $2 / 3$ do valor da DTPD da residência dos sujeitos (DTPD casa).

\subsection{Covariáveis}

Diversos estudos têm demonstrado o quanto características socioeconômicas e de saúde afetam negativamente a cognição e também estão associadas a viver em áreas mais poluídas (Vormittag et al., 2013; WHO, 2019). Para reduzir o confundimento, selecionamos variáveis que estão diretamente relacionadas à cognição e à exposição aos poluentes do ar baseados em estudos prévios, tais como: idade, gênero, educação, etnia, 
hipertensão, diabetes e doenças cardiovasculares, outras variáveis importantes também foram incluidas em nossa análise, entre elas: tabagismo, etilismo, atividade física, dislipidemia e índice de massa corporal.

\subsection{Análise estatística}

Trata-se de um estudo transversal que pretende analisar a associação entre exposição à poluição atmosférica medida indiretamente pela DTDP e alterações em escores de função cognitiva no estudo ELSA-Brasil. As análises descritivas incluem o cálculo de distribuições de frequência para variáveis dicotômicas e medidas de tendência central e dispersão para variáveis contínuas. Assim, para as variáveis dependentes utilizamos o escore-z dos testes cognitivos que foi calculado para cada um dos testes de cognição (teste de lista de palavras do CERAD, teste de evocação de palavras, teste de reconhecimento de palavras, testes de fluência verbal fonêmico (letra "F") e semântico (animais) e teste detrilha versão B), subtraindo a pontuação do teste de cada participante pela média da pontuação da amostra e dividindo pela diferença do desvio padrão (SD) das pontuações. O escore-z global cognitivo foi calculado pela média do escore-z dos três testes (memória, fluência verbal e teste de trilhas). Assim, um escore-z de -1 descreveria o desempenho cognitivo que é 1 DP abaixo da média do escore do teste. Os escores-z para o TMT foram mulitplicados por -1 , porque escores mais altos nesse teste indicam pior desempenho, enquanto escores mais altos nos outros testes estão relacionados a melhor desempenho. Utilizamos a análise de variância para comparar a média dos desfechos (escores cognitivo) entre as categorias das variáveis de exposição (DTPD). Na análise multivariada utilizamos modelos de regressão linear (desfechos contínuos) para investigar a associação existente entre os desfechos e uma série de covariáveis, categóricas ou contínuas, buscando controlar possiveis variáveis confundidoras. Assim, foram construídos três modelos de análise ajustados, sendo o primeiro modelo ajustado 
por idade e sexo. O segundo modelo também foi ajustado por idade e sexo, além de raça e educação. Já o terceiro modelo foi ajustado pelas variáveis contidas no primeiro e segundo modelo, além das variáveis de tabagismo, atividade física, depressão, uso de álcool, diabetes, LDL-colesterol, doença cardiovascular, hipertensão arterial sistêmica, tempo gasto no mesmo local e horário de sua casa. Os softwares utilizados para essas análises foram o programa Excele o software estatístico STATA versão 14.2, para a análise dos dados. 


\section{ARTIGOS PRODUZIDOS}

\section{POLUENTES DO AR COMO FATOR DE RISCO PARA O DESEMPENHO COGNITIVO E DEMÊNCIA}

Este capítulo apresenta o artigo "Air Pollutants As A Risk Factor For Cognitive Impairment And Dementia” de autoria de Douglas Rene Rocha Silva, Claudia Kimie Suemoto e Nelson Gouveia. O artigo foi publicado na revista Cadernos de Saúde Pública.

Para citação: SILVA DRR, SUEMOTO CK, GOUVEIA N. Air pollutants as a risk factor for cognitive impairment and dementia.Cad. Saúde Pública 2019; 35(8):e00085919. 
Air pollutants as a risk factor for cognitive PERSPECTIVES impairment and dementia

Poluentes do ar como fator de risco para o desempenho cognitivo e demência

Contaminantes atmosféricos como factor de riesgo para el desempeño cognitivo y la demencia

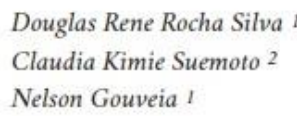

doi: $10.1590 / 0102-311 \times 00085919$

\section{Introduction}

\section{Population aging and cognition}

Numerous advances in the last 100 years, principally in access to new technologies, goods and services, medical care, and expanded availability of medicines, have led to a major increase in the population's life expectancy worldwide. This has made population aging one of the most important global transformations in human life. However, some nations, especially low and middle-income countries, will experience this increase in life expectancy (and consequently in the elderly population) at an unprecedented pace when compared to the same phenomenon in Europe and North America. These countries will thus have to prepare quickly to deal with a growing burden of highly common diseases at more advanced ages 1.

Currently, an estimated $12 \%$ of the global population is over 60 years of age, and projections show that the elderly will represent $21.5 \%$ of the world population by the end of this century. It will be the first time in human history that there will be more old people than young people 2. In this context, cognitive aging and the diseases resulting from this process pose a major challenge for healthy aging. Importantly, normal cognitive aging involves a natural and dynamic process that includes modifications in biological functioning (molecular, cellular, histological, and organic), psychiatric functioning (cognitive and psycho-affective dimensions), and social functioning 3.4 .5 . Healthy aging can thus be accompanied by a certain decline in cognitive reserves, but which does not interfere substantially in daily activities. In other cases, when cognitive aging is not successful, there is a sharper decline in cognition, which may be linked to factors extrinsic to the individual, such as sedentary 
lifestyle, smoking, low schooling, alcohol and drug consumption, and exposure to air pollution.

\section{Air pollutants and human health}

In the last 40 years, studies on the impacts of air pollutants, especially in cities, are unanimous in associating greater length of individual exposure to air pollutants with elevated risk of developing inflammatory processes, diseases of the respiratory system, and cardiovascular problems. In most cases, the elderly and children are the most susceptible to developing these diseases. According to recommendations by the World Health Organization (WHO), various countries have adopted more rigorous limits on the concentration of air pollutants. However, even today, $91 \%$ of the world population lives in cities where the air quality exceeds the limits suggested by the WHO 2. As a consequence, in $2016,8 \%$ of all deaths in the world were attributed to exposure to air pollutants, especially in large urban areas, where the main source of emissions is motor vehicle traffic. Collectively, air pollutants consist of a complex and diverse mixture of chemical substances with the capacity to cause harm or discomfort to humans. Such pollutants are thus now the second leading cause of deaths from non- communicable diseases, after smoking, whereas the latter can be avoided by the individual, unlike exposure to environmental air pollutants. In Brazil, despite the data's limitations, an estimated 66,000 yearly deaths are attributed to exposure to air pollutants annually 2. However, this number may be even higher, since projections for the next 15 years only for the State of São Paulo state that if nothing is done, exposure to air pollutants will be the cause of 250 thousand deaths, one million hospitalizations at a cost of 1.5 billion BRL only in the state of São Paulo 6,7.

In relation to the harmful effects of air pollution on cognitive performance, a cohort study of 2.1 million persons in Ontario, Canada, showed a positive association between fine particulate matter $\left(\mathrm{PM}_{2.5}\right)$, even at low concentrations, and incidence of dementia 8. In Sweden, a study of 1,806 adult volunteers found a positive association between air pollutants and increased incidence of dementia and Alzheimer's disease 9. In Taiwan, two cohort studies, the first with 96,000 and the second with 30,000 volunteers, found that exposure to the pollutants nitrogen dioxide $\left(\mathrm{NO}_{2}\right)$, fine particulate matter $\left(\mathrm{PM}_{2.5}\right)$, ozone $\left(\mathrm{O}_{3}\right)$, and carbon monoxide ( $\mathrm{CO}$ ) was also associated with increased incidence of dementia 10,11. Taken together, these studies suggest that prolonged exposure to air pollutants, especially those produced by motor vehicle traffic in large cities, like particulate matter, show a positive association with cognitive decline and increased incidence of dementia 12,13. This association is biologically plausible, because once inside the body, these pollutants can trigger chronic and systemic inflammation, besides directly harming the nervous system 14,15,16,17,18,19.

Finally, it is important to recall that before individuals present dementia per se, a smoldering process of cognitive impairment occurs, which is not 
sufficiently serious in the beginning to lead to functional loss and interfere in quality of life. However, this cognitive impairment is of great importance for early diagnosis of risk factors for dementia at more later ages 1,20.

\section{Timeliness of the study}

Brazil and its Latin American neighbors are experiencing an accelerated and irreversible process of population aging. In addition, many countries have cities whose air pollutant emissions fail to comply with the WHO guidelines 2. Meanwhile, according to forecasts for $2030,63 \%$ of the world population with dementia will be living in low and middle-income countries 1 . Thus, studies that seek to identify and understand possible environmental risk factors and their influence on the pathological cognitive aging process in young and middle-aged adults are highly important for avoiding such outcomes in the future. Research on chronic exposure to air pollutants and their role in neurodegenerative processes in young and elderly populations take on great importance in a scenario of growing life expectancy and high emissions of air pollutants.

\section{Final remarks}

The relationship between exposure to air pollutants and neurodegenerative outcomes is still not fully established, but it is already considered a global concern. Many countries, including most of the Latin American nations, still lack data and studies that seek to understand this association in their realities, which indicates a relevant gap. In the coming years, Brazil and other Latin American countries should thus make progress in their understanding of the association between air pollutants and cognitive outcomes, aimed at minimizing the impact of this association in order to provide individuals with healthier aging in urban settings.

\footnotetext{
1. National Institute of Health; National Institute of Neurological Disorders and Stroke; National Institute on Aging. The dementias: hope through research. Bethesda: National Institute of Health; 2013.

2. World Health Organization. Ambient air pollution - a major threat to health and climate. Copenhagen: World Health Organization; 2018.

3. Brito FC, Litvoc CJ. Conceitos básicos. In: Brito FC, Litvoc CJ, organizadores. Envelheci- mento - prevenção e promoção de saúde. São Paulo: Atheneu; 2004. p. 1-16.

4. Fechine BRA, Trompieri N. O processo de envelhecimento: as principais alterações que acontecem com oidoso com o passar dos anos. InterSciencePlace - Revista Científica Inter- nacional 2012; 1:106-94.

5. Günther IA. Envelhecimento, relações sociais e ambiente. In: Falcão DVS, Araújo LF, orga- nizadores. Psicologia do envelhecimento. São Paulo: Alínea; 2011. p. 11-25.

6. Vormittag EMPA, Rodrigues CG, Miranda MJ, Cavalcante JA, Costa RR, Camargo CA, et al. Avaliação do impacto da poluição atmosférica no Estado de São Paulo sob a visão da saúde. São Paulo: Instituto Saúde e Sustentabilidade; 2013.

7. Vormittag EMPA, Saldiva PHN. Qualidade do ar no Estado de São Paulo 2017. Sob a visão da saúde. São Paulo: Instituto Saúde e Sustentabi- lidade; 2013.

8. Chen H, Kwong JC, Copes R, Hystad P, van Donkelaar A, Tu K, et al. Exposure to ambient air pollution and the incidence of dementia: a population-based cohort study. Environ Int 2017; 108:271-7.

9. Oudin A, Forsberg B, Adolfsson AN, Lind N, Modig L, Nordin M, et al. Traffic-related air pollution and dementia incidence in northern Sweden: a longitudinal study. Environ Health Perspect 2016; 124:306-12.

10. Chang KH, Chang MY, Muo CH, Wu TN, Chen CY, Kao CH. Increased risk of dementia in patients exposed to nitrogen dioxide and carbon monoxide: a population-based retrospective cohort study. PLoS One 2014; 9:e103078.
} 
11. Jung CR, Lin YT, Hwang BF. Ozone, particulate matter, and newly diagnosed Alzheimer's disease: a population-based cohort study in Taiwan. J Alzheimers Dis 2015; 44:573-84.

12. Block ML, Calderón-Garcidueñas L. Air pollution: mechanisms of neuroinflammation and CNS disease. Trends Neurosci 2009; 32:506- 16

13. Calderón-Garcidueñas L, Reed W, Maronpot RR, Henríquez-Roldán C, Delgado-Chavez R, Calderón-Garcidueñas A, et al. Brain inflam- mation and Alzheimer's-like pathology in individuals exposed to severe air pollution. Toxi- cologic Pathology 2004; 32:650-8.

14. Calderón-Garcidueñas L, Azzarelli B, Acuna H, Garcia R, Gambling TM, Osnaya N, et al. Air pollution and brain damage. Toxicologic Pathology 2002; 30:373-89.

15. Calderón-Garcidueñas L, Mora-Tiscareño A, Ontiveros E, Gómez-Garza G, Barragán-Mejía G, Broadway J, et al. Air pollution, cognitive deficits and brain abnormalities: a pilot study with children and dogs. Brain Cogn 2008; 68:117-27.

16. Tuppo E, Arias H. The role of inflammation in Alzheimer's disease. Int J Biochem Cell Biol 2005; 37:289-305.

17. Nunomura A, Castellani RJ, Zhu X, Moreira PI, Perry G, Smith MA. Involvement of oxidative stress in Alzheimer disease. J Neuropathol 2006; 65:631-41.

18. Genc S, Zadeoglulari Z, Fuss SH, Genc K. The adverse effects of air pollution on the nervous system. J Toxicol 2012; 2012:782462.

19. Numan M, Brown J, Michou L. Impact of air pollutants on oxidative stress in common autophagy -mediated aging diseases. Int J Environ Res Public Health 2015; 12:2289-205.

20. Burlá C, Camarano AA, Kanso S, Fernandes D, Nunes R. Panorama prospectivo das demên- cias no Brasil: um enfoque demográfico. Ciênc Saúde Colet 2013; 18:2949-56. 


\section{DENSIDADE DE TRÁFEGO VEICULAR E DESEMPENHO COGNITIVO NO}

ESTUDO ELSA-BRASIL

Este capítulo apresenta o artigo "Vehicular traffic density and cognitive performance in the ELSA-Brasil study" de autoria de Douglas Rene Rocha Silva, Claudia K. Suemoto, Itamar de Souza Santos, Paulo A. Lotufo, Isabela Benseñor, Nelson Gouveia. O artigo foi submetido para piblicação na revista Environmental Research.

Para citação: Silva, DRR, Suemoto CK, Santos IS, Lotufo PA, Benseñor I, Gouveia N. Vehicular traffic density and cognitive performance in the ELSA-Brasil study. submitted. 


\section{Vehicular traffic density and cognitive performance in the ELSA-Brasil study}

Douglas Rocha (1), Claudia K. Suemoto (2,3), Itamar de Souza Santos (2), Paulo A.

Lotufo (2), Isabela Benseñor (2), Nelson Gouveia (1)

(1) Department of Preventive Medicine, University of São Paulo Medical School, São Paulo, SP, Brazil.

(2) Center for Clinical and Epidemiological Research, University of São Paulo, São Paulo, SP, Brazil.

(3) Division of Geriatrics, University of São Paulo Medical School, São Paulo, SP, Brazil. 


\begin{abstract}
Background: Despite the knowledge about the deleterious effects of air pollutants and their influence on mortality and morbidity due to respiratory and cardiovascular diseases, little is known about the relationship between atmospheric pollutants and neurological diseases. Recently, studies from high income countries have suggested an association between exposures to air pollutants with cognitive impairment. Thus, we investigated the association of air pollution with cognitive performance in the participants of the Brazilian Longitudinal Study of Adult Health (ELSA-Brasil).
\end{abstract}

Methods: Cognitive function was evaluated using the word list, the verbal fluency, and the trail making tests (TMT). Pollutant exposure was evaluated indirectly using the distance-weighted traffic density (DWTD) of participants' residence and workplace. We investigated the cross-sectional association between DTWD and cognitive test scores using adjusted linear regression models for sociodemographic and clinical variables. We excluded participants with incomplete data for the cognitive tests or when indicators of exposure to ambient air pollution were unavailable. Participants with a self-reported medical diagnosis of stroke at baseline and participants who were using medications that indicate the presence of active neurologic or psychiatric diseases were also excluded.

Results: Among 5,061participants from the São Paulo center, 3,050 were included (mean age $=52.1 \pm 9.2$ years, 56,5\% women, and $63,6 \%$ white). In the simple linear regression models, participants in the higher tertile of combined DWTD (residence and workplace) presented better cognitive performance in all tests when compared to participants in the lower tertile, except in the TMT test. However, DWTD was not associated with cognitive performance in adjusted linear models especially when adjusted for socioeconomic variables (education and race). We found similar results when we investigated the association of cognitive performance with DTWD near participants' workplace and 
residence separately.

Conclusion: Air pollutants were not associated with worse cognitive performance in a large sample of middle-aged and older adults.

\section{Introduction}

The number of people with dementia worldwide was estimated to be 46.8 million in 2015 . This number is projected to double every 20 years, reaching 75 million in 2030, and 131.5 million cases in 2050, if the current trend of a new case every three seconds is maintained (WAR 2015; WHO 2017). Dementia occurs frequently in older adults and it is caused by a wide variety of brain diseases that gradually lead to a decrease in cognitive performance, which is severe enough to interfere with daily activities (Forlenza et al. 2013). However, neuropathological lesions can be found in middle-aged individuals, and they can progress asymptomatically for 20 to 30 years before the first clinical symptoms of dementia (Tuokko et al. 2003). A number of factors have been associated with cognitive impairment, such as aging (Ferri et al. 2012, Nitrini et al., 2004), cardiovascular risk factors (WAR 2014; Duron et al. 2008), genetic factors, and low educational attainment (NIH 2013).

Currently, increasing evidence places chronic exposure to urban air pollutants as a risk factor for cognitive impairment and dementia. This association was demonstrated by Chen et al. (2017) in a large cohort in Canada, by monitoring 2.1 million people living in the Ontário city between 2001 and 2013. In that study, 6.1\% of dementia cases were attributed to exposure to fine particulate matter $\left(\mathrm{PM}_{2.5}\right)$ and nitrogen dioxide (NO2). In Sweden, a cohort study that followed 1,806 volunteers for 15 years found an increased risk for vascular dementia and Alzheimer's disease in volunteers who were chronically exposed to air pollution (Oudin et al. 2016). Finally, the results of a recent systematic review suggested that exposure to a wide range of air pollutants emitted by vehicular 
traffic has been associated with worse cognitive performance since childhood, but mainly affecting older adults (Clifford et al. 2016).

The prevalence of dementia cases has substantially increased in low- and middle-income countries (LMIC). In 2015, 58\% of people with dementia lived in LMIC and projections for 2050 estimate that this rate will increase to 68\% (WAR 2015). Urban levels of air pollutants follow the same path. Today, despite efforts to reduce the emission of air pollutants 9 out of 10 people breathe polluted air (WHO 2018). Although most of these people live currently in LMIC, there is no study so far that has investigated the association between urban air pollutants and cognitive performance in these countries, where control of air quality standards is an important public health issue (Parra et al. 2018; Prince et al. 2013; Nitrini et al. 2009). Thus, examining cognitive performance in middle aged adults exposed to urban air pollutants can be an important step for detecting individuals who are at a higher risk of cognitive impairment and dementia. This study aims to investigate the cross-sectional association between traffic density and cognitive performance in adult volunteers from a large cohort study in Latin America.

\section{Material and Methods}

\section{Participants}

This a cross-sectional analysis from the Brazilian Longitudinal Study on Adult Health (ELSA-Brasil), which is a cohort of 15,105 civil servants living in six Brazilian cities (Belo Horizonte, Vitoria, Salvador, São Paulo, Rio de Janeiro, and Porto Alegre). Participants were between 35 and 74 years old and were free of dementia at enrollment (August 2008 to December 2010). Baseline assessment included information on sociodemographic, clinical history, lifestyle factors, mental health, occupational exposure, and family history of diseases. We also performed anthropometric measures, and laboratory and imaging exams. A detailed description of the ELSA-Brasil study can 
be found elsewhere (Aquino et al. 2012; Schmidt et al. 2014). The present study is restricted only to participants from São Paulo, where indicators of exposure to ambient vehicular traffic pollutants were available (Figure 1). We excluded participants with a self-reported medical diagnosis of stroke at baseline, in order to minimize the effect of previous cerebrovascular disease on cognitive function. We also excluded participants who were using medications that indicate the presence of active neurologic or psychiatric diseases, or that could directly interfere with performance in the cognitive tests (antiparkinsonian agents, antipsychotic medications, and anticonvulsants). Finally, we excluded participants with incomplete data for the cognitive tests or when indicators of exposure to ambient air pollution were unavailable. The study was approved by the local institutional review boards, and all participants signed an informed consent at the time of enrollment.

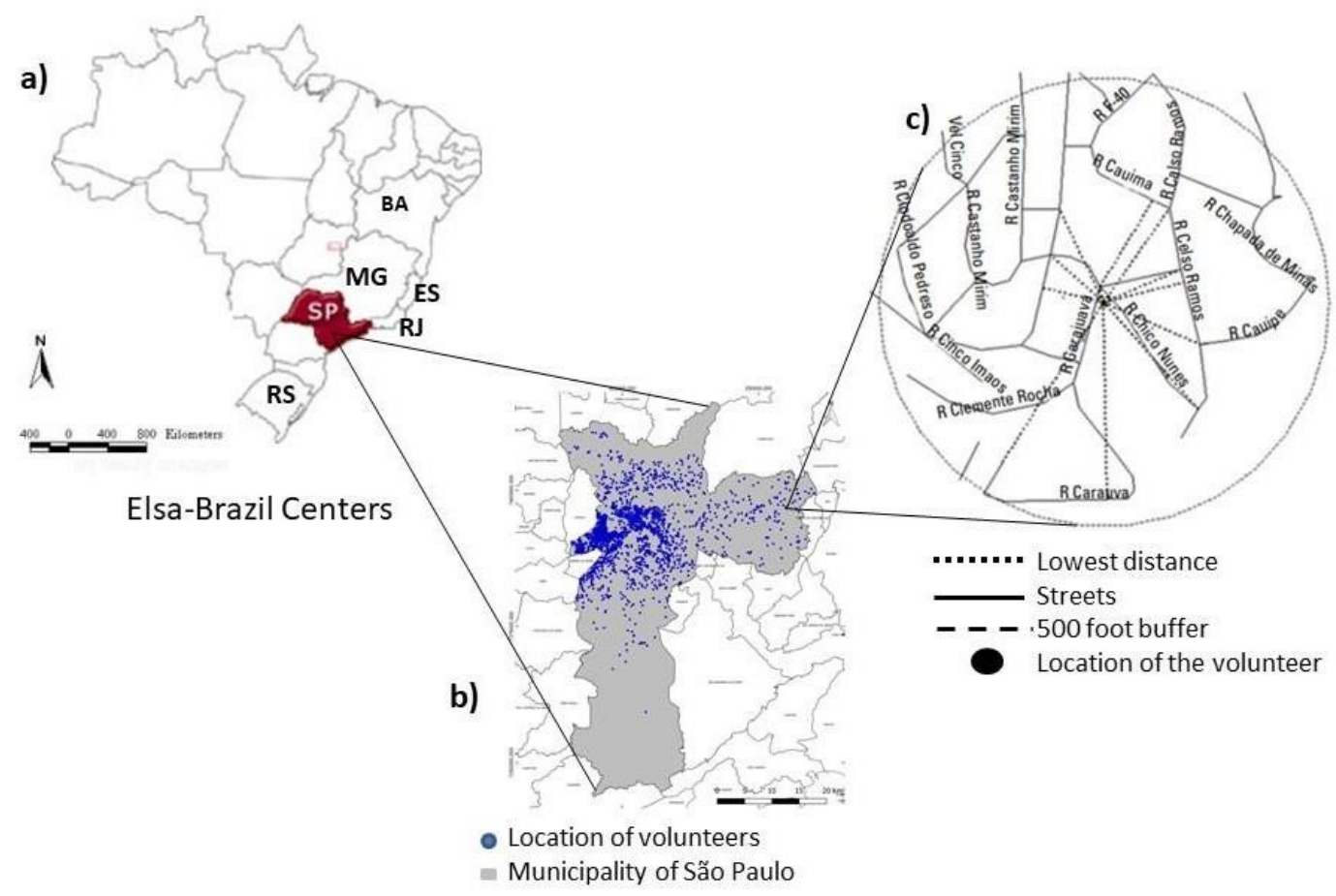

Figure 1. (A) ELSA-Brasil study centers; (B) Geolocation of the study volunteers; (C) Example of a region buffer of one volunteer. 


\section{Cognitive Function Evaluation}

Cognitive function was assessed using the following tests: (a) the Brazilian version of the Consortium to Establish a Registry for Alzheimer's Disease Word List Memory Test (CERAD-WLMT) (Morris et al. 1989; Bertolucci et al. 2001); (b) the Semantic Verbal Fluency Test (SVFT); (c) the Phonemic Verbal Fluency Test (PVFT) (Bertolucci et al. 1998; Nitrini et al. 2004 ); and (d) the Trail Making Test version B (TMT) (Greenlief et al. 1985). Trained examiners administered the tests in a fixed order during a single session, in a quiet room with good lighting, and low levels of noise or other distractions (Passos et al. 2014). The CERAD-WLMT is a test of verbal learning and memory. Participants were asked to read and learn 10 words after three exposures to the word list. After a 5min filled delay, the participants were given 60s to record these words. The score was equal to the number of recalled words. The verbal fluency tests evaluate language and executive function. Participants were asked to name as many animals as possible. The score was equal to the total number of recorded words in one minute. In the PVFT, participants were asked to generate as many words as possible beginning with the letter $\mathrm{F}$ to assess language and executive function. The score in this test was the total number of words generated during one minute (Shao et al. 2014). The TMT is a test of executive function, processing speed, and attention. The participants were instructed to draw lines connecting letters and numbers in an order that alternated between increasing numeric values and alphabetic order (A, 1, B, 2, C, 3, D...). The test score was the total time taken to complete the task, in seconds. To facilitate comparisons across cognitive tests, we generated z-scores by subtracting the participant's score in each test from the sample mean score and dividing the difference by the sample standard deviation (SD) (Rawlings et al. 2014; Bennett et al. 2012). We combined the three scores in the CERAD- WLMT (i.e., immediate recall, delayed recall, and recognition) by averaging the z-scores 
for each memory test and then standardizing this averaged score. We also combined the verbal fluency test scores (SVFT and PVFT) by averaging the z-scores for each test and standardizing the mean. For the test TMT we inverted the z-score signal because higher scores indicate poorer performance in the original TMT, whereas higher scores on the other tests are related to better performance. Therefore, for all domains, a z-score of -1 describes a cognitive performance that is $1 \mathrm{SD}$ worse than the mean sample performance. Finally, we averaged and standardized the z-scores of all tests as a measure of composite global cognitive score (Suemoto et al. 2018).

\section{Traffic Density Weighted by Distance}

To estimate the exposure to air pollutants for each participant, we used an indirect indicator related to traffic, the Distance Weighted Traffic Density (DWTD). This measure assumes that vehicular emissions on the roads approach a Gaussian distribution and that $96 \%$ of pollutants disperse up to 150 meters from the center of the road (Pearson et al. 2000; Habermann et al. 2011). Information about the road network and vehicular traffic in 2007 was used, since it was the closest assessment available to the time of enrollment in the ELSA-Brasil study. This information included the cartographic base of the roads, number of scrolling bands, and maximum speed allowed in the segment provided by the Department of Transport of the city of São Paulo [Companhia de Engenharia de Trafego

(CET)]. This contained a simulation of traffic flows on every segment of all freeways, arterials, and collector roads attained from systematic counts conducted in the peak hours. This dataset also contained an estimate of the total traffic flow from all local roads inside predefined microareas (polygons). For local roads without information on flows, we followed a procedure developed by CET that divides the estimated total flow for each polygon proportionally for each local road according to its length inside the area. We performed this procedure using Maptitude software (version 4.6; Calliper Corp., Newton, 
MA, USA), which enabled us to attribute traffic flow values for all local roads without counts. Using a similar method to that of Pearson et al. (2000), we calculate the DWTD for the residential and working address of each participant. First, we delineated a radius of $156,5 \mathrm{~m}$ (500 feet) around the address of each participant and identified all streets contained in this buffer for later calculation of the perpendicular distance between each street and the address (Figure 1c). We then gathered the weighted values of the traffic flow for all streets surrounding the subject's residence to compose the DWTD of each participant in the study (Habermann et al. 2011). For the calculation of the DWTD that combines the density oftraffic of the residence and workplace, we considered 8 hours as the average time of permanence of the employees in their respective work units. The remaining hours of the day (16 hours) were attributed to activities at home. Therefore, the combined DWTD calculation was performed with the weighted average of $1 / 3$ of the DWTD value of the workplace and 2/3 of the DWTD value of the subjects' residence.

\section{Other Measurements}

Information on sociodemographic and clinical variables included age, sex, race, education, marital status, hypertension, diabetes, body mass index, cardiovascular disease, low density lipoprotein-cholesterol (LDL), high density lipoprotein-cholesterol (HDL), smoking, alcohol use, leisure time physical activity, and depression. Education was classified in less than elementary school, middle school, high school, and college degree or higher. Diabetes was defined as the presence of one of the following: medical history of diabetes, use of medication to treat diabetes, fasting plasma glucose $\geq 126$ $\mathrm{mg} / \mathrm{dL}$ and 2-h glucose post load test $\geq 200 \mathrm{mg} / \mathrm{dL}$. LDL cholesterol was estimated using the Friedewald equation and, when the triglyceride (TG) values were greater than 400 $\mathrm{mg} / \mathrm{dL}$, the dosage was determined by the homogeneous colorimetric enzymatic method without precipitation. HDL cholesterol dosing was performed using the homogeneous 
colorimetric method without precipitation (Schmidt et al. 2013). Hypertension was defined using at least one of three criteria: systolic blood pressure $\geq 140 \mathrm{mmHg}$, diastolic blood pressure $\geq 90 \mathrm{mmHg}$, or use of antihypertensive medication. Cardiovascular disease was considered when participants reported previous diagnosis of myocardial infarction, myocardial revascularization, or heart failure. Alcohol use and smoking were categorized in never, former, and current user. Physical activity was categorized into three groups: no physical activity, leisure activity $\leq 150$ min per week, and leisure activity $>150$ min per week. Weight and height were measured in participants with light clothing and without shoes, and body mass index was calculated dividing the weight in $\mathrm{kg}$ by the square of the height in meters. The diagnosis of depression was based on the International Classification of Diseases (ICD-10) criteria (WHO 2004) and used the participant's responses to the Clinical Interview Schedule Revised (Lewis et al. 1992).

\section{Statistical Analysis}

Descriptive analyses included the calculation of frequency distributions for categorical variables and measures of central tendency and dispersion for continuous variables. We used the z-scores of the cognitive tests as dependent variables. We used one-way ANOVA for continuous variables and chi-square test for categorical ones to compare these variables by tertiles of DWTD. We used hierarchical linear regression models to investigate the association between cognitive test z-scores and DWTD adjusted for possible confounding variables. The first model was adjusted for age and sex. The second model was additionally adjusted for age, sex, race, and education. The third model was adjusted for the variables included in the previous models, and for hypertension, diabetes, LDL cholesterol, HDL cholesterol, cardiovascular disease, smoking, alcohol use, physical activity, depression, and time (in years) that the participants lived in their residence and worked at their institutions. We used Excel to calculate the DTWD 
variables, and the software STATA version 14.2 (StataCorp, College Station, TX) for statistical analysis.

\section{Results}

Among 15,105 participants, 5,061 were from the São Paulo center and eligible to participate in the study. After applying the study criteria, 3,050 participants were included (Figure 2).

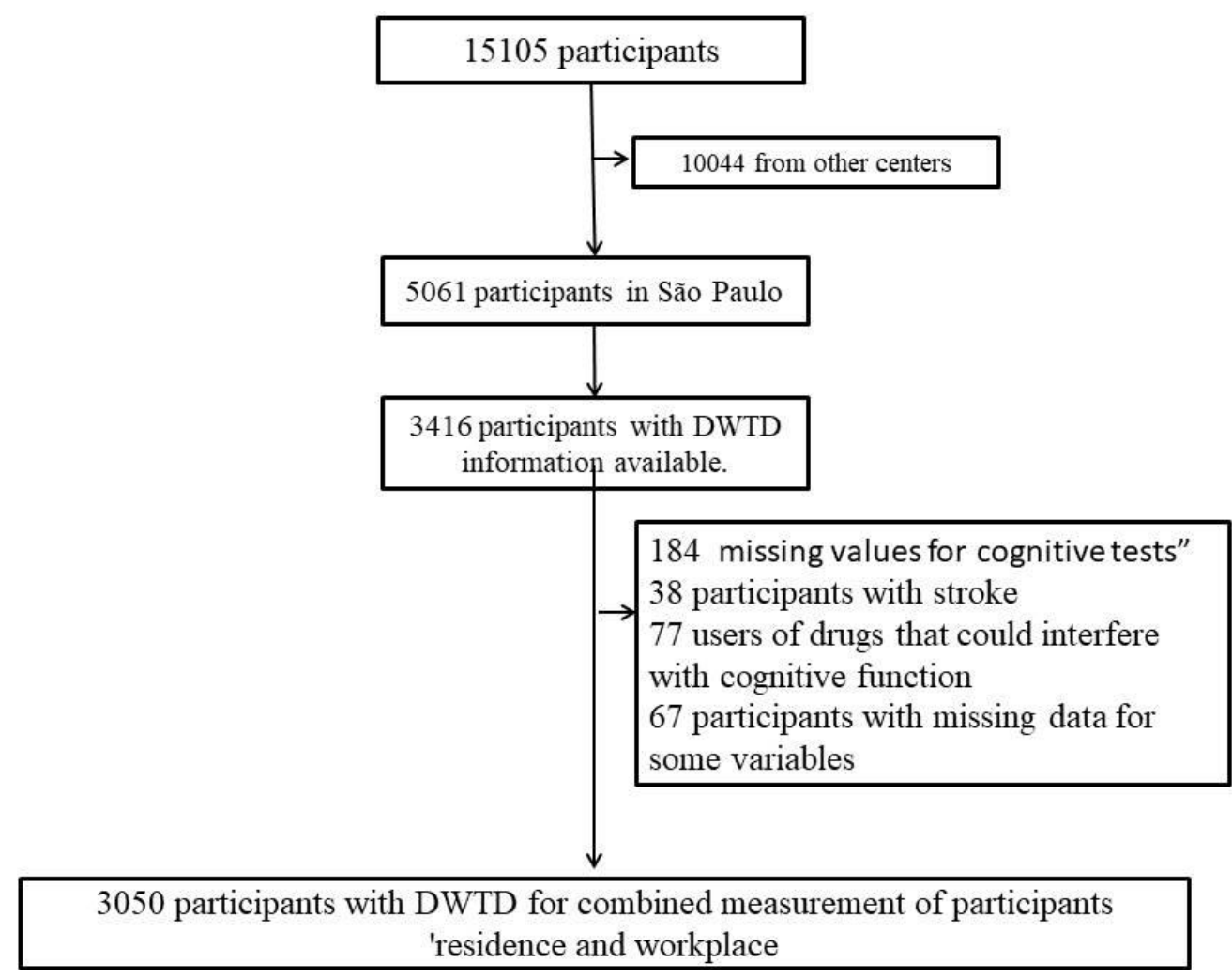

Figure 2. Flowchart of study participants.

The mean age of the sample was $52.1 \pm 9.2$ years, $43.5 \%$ were male, $63.6 \%$ were white, and $54.1 \%$ had college education or more. Hypertension was present in $31.6 \%$ of the sample, $72.0 \%$ used alcohol, $53.4 \%$ never smoked, and $76.0 \%$ were physically inactive. Participants recorded an average of $20.3 \pm 3.8$ words in the CERAD immediate word recall, $6.6 \pm 1.9$ words in the CERAD delayed word recall, $9.5 \pm 0.8$ words in the CERAD word recognition, $18.2 \pm 5.0$ words in the SVFT, $12.5 \pm 4.4$ in the PVFT, and they 
completed the TMT in $119.5 \pm 84.9$ seconds. (Supplementary Table 1).

Table 1 shows the association of DWTD with sociodemographic and clinical variables.

DWTD was associated with age, sex, marital status, race, education, alcohol use, immediate word recall test, SVFT, and TMT.

Association of Distance Weighted Traffic Density (DWTD) with sociodemographic and clinical variables $(n=3,050)$

\begin{tabular}{|c|c|c|c|c|}
\hline Variable & 1st Tertile & 2nd Tertile & 3rd Tertile & $P$-value \\
\hline $\begin{array}{l}\text { Immediate word recall }{ }^{\mathrm{a}} \text {, } \\
\text { mean }(\mathrm{SD})^{\mathrm{b}}\end{array}$ & $-0.48(0.9)$ & $0.01(1.0)$ & $0.16(0.9)$ & $<0.0001$ \\
\hline $\begin{array}{l}\text { Delayed word recall }{ }^{\mathrm{a}} \text {, } \\
\text { mean }(\mathrm{SD})^{\mathrm{b}}\end{array}$ & $0.13(0.9)$ & $0.04(09)$ & $0.10(0.9)$ & 0.184 \\
\hline $\begin{array}{l}\text { Word recognition }{ }^{\mathrm{a}} \\
\text { mean }(\mathrm{SD})^{\mathrm{b}}\end{array}$ & $0.03(0.9)$ & $0.05(1.0)$ & $0.04(0.9)$ & 0.485 \\
\hline $\begin{array}{l}\text { Verbal fluency (animal) } \\
\text { mean }(S D)^{\mathrm{b}}\end{array}$ & $-0.09(0.9)$ & $0.06(0.9)$ & $0.19(1.0)$ & $<0.0001$ \\
\hline $\begin{array}{l}\text { Verbal fluency (letter F) } \\
\text { mean }(S D)^{\mathrm{b}}\end{array}$ & $0.03(0.9)$ & $0.08(0.9)$ & $0.13(0.9)$ & 0.184 \\
\hline $\begin{array}{l}\text { Trail making test (seconds), } \\
\text { mean (SD) }\end{array}$ & $-0.03(1.0)$ & $0.01(0.9)$ & $0.16(0.8)$ & $<0.0001$ \\
\hline Age (years), mean $(\mathrm{SD})^{\mathrm{b}}$ & $49.5(7.9)$ & $51.2(9.2)$ & $53.5(9.2)$ & $<0.0001$ \\
\hline Sex, $\%^{c}$ & & & & 0.003 \\
\hline Female & 49.47 & 54.86 & 59.31 & \\
\hline Marital, $\%^{\mathrm{c}}$ & & & & $<0.0001$ \\
\hline Married & 73.5 & 67.5 & 61.9 & \\
\hline Separate & 13.4 & 15.1 & 14.9 & \\
\hline Single & 6.01 & 12.0 & 15.3 & \\
\hline Widower & 3.89 & 2.89 & 4.72 & \\
\hline Other & 3.18 & 2.45 & 3.03 & \\
\hline Race, $\%^{c}$ & & & & $<0.0001$ \\
\hline Black & 17.3 & 12.6 & 9.24 & \\
\hline Brown & 27.9 & 19.6 & 15.1 & \\
\hline White & 50.5 & 60.7 & 68.9 & \\
\hline Asian & 3.89 & 5.93 & 5.78 & \\
\hline Indigenous & 0.35 & 1.04 & 0.92 & \\
\hline Education, $\%{ }^{c}$ & & & & $<0.0001$ \\
\hline $\begin{array}{l}\text { Less than Elementary } \\
\text { school }\end{array}$ & 7.77 & 5.78 & 3.81 & \\
\hline Less than middle school & 7.07 & 7.12 & 5.15 & \\
\hline Complete high school & 50.8 & 37.2 & 28.9 & \\
\hline University degree & 34.2 & 49.9 & 62.02 & \\
\hline Diabetes, $\%^{\mathrm{c}}$ & & & & 0.923 \\
\hline Yes & 20.1 & 20.01 & 19.46 & \\
\hline Hypertension, $\%$ & & & & 0.686 \\
\hline Yes & 31.8 & 30.8 & 32.7 & \\
\hline $\begin{array}{l}\text { LDL-cholesterol (mg/dl), mean } \\
(\mathrm{SD})^{\mathrm{b}}\end{array}$ & $132.5(37.14)$ & $128.7(33.2)$ & $130.8(33.6)$ & 0.184 \\
\hline $\begin{array}{l}\text { HDL-cholesterol (mg/dl), mean } \\
(\mathrm{SD})^{\mathrm{b}}\end{array}$ & $55.0(13.9)$ & $56.5(13.9)$ & $58.0(14.8)$ & 0.0007 \\
\hline Alcohol use, $\%^{\mathrm{c}}$ & & & & 0.01 \\
\hline Never & 11.3 & 10.9 & 9.8 & \\
\hline Former & 22.6 & 18.6 & 15.5 & \\
\hline Current & 66.0 & 70.4 & 74.6 & \\
\hline Smoking, $\%^{\mathrm{c}}$ & & & & 0.03 \\
\hline Never & 55.4 & 51.9 & 54.5 & \\
\hline Former & 27.9 & 29.8 & 31.6 & \\
\hline Current & 16.6 & 18.2 & 13.9 & \\
\hline Physical activity, $\%^{\mathrm{c}}$ & & & & 0.228 \\
\hline No & 79.1 & 77.1 & 74.3 & \\
\hline Yes $<150 \mathrm{~min} / \mathrm{wk}$ & 11.6 & 13.8 & 14.7 & \\
\hline
\end{tabular}




\begin{tabular}{|c|c|c|c|c|}
\hline$Y e s, \geq 150 \mathrm{~min} / \mathrm{wk}$ & 9.19 & 8.97 & 10.9 & \\
\hline $\begin{array}{l}\text { Body mass index }\left(\mathrm{Kg} / \mathrm{m}^{2}\right) \text {, } \\
\text { mean }(\mathrm{SD})^{\mathrm{b}}\end{array}$ & $27.1(4.5)$ & $26.9(4.7)$ & $27.2(5.0)$ & 0.162 \\
\hline Depression, $\%^{c}$ & & & & 0.815 \\
\hline No & 96.1 & 96.4 & 95.9 & \\
\hline Yes & 3.89 & 3.56 & 4.02 & \\
\hline Cardiovascular disease, $\mathrm{n}(\%)$ & & & & 0.890 \\
\hline No & 97.17 & 97.41 & 97.11 & \\
\hline Yes & 2.83 & 2.59 & 2.89 & \\
\hline
\end{tabular}

In the simple linear regression models, participants in the higher tertile of combined DWTD (residence and work) presented better global $(\mathrm{p}<0.0001)$, memory $(\mathrm{p}<0.007)$, and verbal fluency performance $(\mathrm{p}<0.001)$ when compared to participants in the lower tertile. On the other hand, participants in the higher tertile of DWTD had worse cognitive performance in the TMT when compared to participants in the lower tertile $(\mathrm{p}<0.0001)$. However, we did not find associations between DTWD and cognitive performance in adjusted models (Table 2). When we investigated the association of cognitive performance with work and home DTWD, we found similar results (Supplementary Tables 2 and 3). 
Table 2 Association of cognitive performance with the Traffic Density Weighted by Distance (DWTD) of the combined measurements of participants' residence and workplace $(n=3,050)$

\begin{tabular}{|c|c|c|c|c|c|c|c|c|c|}
\hline \multirow[b]{2}{*}{ z-Score } & \multirow[b]{2}{*}{ Tertile } & Crude & \multirow[b]{2}{*}{$p$} & Model 1 & \multirow[b]{2}{*}{$P$} & Model 2 & \multirow[b]{2}{*}{$p$} & Model 3 & \multirow[b]{2}{*}{$p$} \\
\hline & & $\beta(95 \% \mathrm{CI})$ & & $\beta(95 \% C I)$ & & $\beta(95 \% C I)$ & & $\beta(95 \% C I)$ & \\
\hline \multirow{2}{*}{$\begin{array}{c}\text { Composite } \\
\text { global }\end{array}$} & 2nd & $0.077(-0.042 ; 0.197)$ & $<0.0001$ & $0.097(-0.018 ; 0.212)$ & $<0.0001$ & $-0.033(-0.131 ; 0.064)$ & 0.07 & $0.035(-0.13 ; 0.063)$ & 0.10 \\
\hline & $3 \mathrm{rd}$ & $0.220(0.101 ; 0.339)$ & & $0.272(0.156 ; 0.388)$ & & $0.032(-0.066 ; 0.131)$ & & $0.027(-0.071 ; 0.126)$ & \\
\hline \multirow{2}{*}{ Memory } & 2nd & $0.025(-0.098 ; 0.149)$ & 0.007 & $0.042(-0.075 ; 0.160)$ & $<0.0001$ & $-0.036(-0.148 ; 0.075)$ & 0.11 & $-0.034(-0.146 ; 0.778)$ & 0.13 \\
\hline & $3 \mathrm{rd}$ & $0.129(0.006 ; 0.252)$ & & $0.178(0.060 ; 0.296)$ & & $0.033(-0.079 ; 0.146)$ & & $0.032(-0.081 ; 0.145)$ & \\
\hline \multirow{2}{*}{$\begin{array}{c}\text { Verbal } \\
\text { fluency }\end{array}$} & 2nd & $0.121(-0.004 ; 0.247)$ & $<0.0001$ & $0.128(0.003 ; 0.254)$ & $<0.0001$ & $0.005(-0.106 ; 0.118)$ & 0.81 & $0.002(-0.110 ; 0.114)$ & 0.86 \\
\hline & $3 \mathrm{rd}$ & $0.230(0.105 ; 0.355)$ & & $0.250(0.124 ; 0.376)$ & & $0.025(-0.088 ; 0.139)$ & & $0.018(-0.094 ; 0.132)$ & \\
\hline \multirow{2}{*}{$\begin{array}{c}\text { Trail } \\
\text { making }\end{array}$} & 2nd & $0.049(-0.070 ; 0.169)$ & $<0.0001$ & $0.075(-0.042 ; 0.193)$ & $<0.0001$ & $-0.056(-0.153 ; 0.039)$ & 0.05 & $-0.061(-0.157 ; 0.034)$ & 0.07 \\
\hline & $3 \mathrm{rd}$ & $0.196(0.076 ; 0.315)$ & & $0.257(0.138 ; 0.375)$ & & $-0.009(-0.087 ; 0.107)$ & & $0.001(-0.096 ; 0.097)$ & \\
\hline
\end{tabular}

Model 1: Linear regression model adjusted for age and sex.

Model 2: Linear regression model adjusted for age, sex, race, and education.

Model 3: Linear regression model adjusted for age, sex, race, education, LDL-cholesterol, HDL-cholesterol, hypertension, diabetes, cardiovascular disease, smoking, body mass index, alcohol use, physical activity, depression, and number of years living in the current residency.

Reference: $1^{\text {st }}$ tertile of DWTD 


\section{Discussion}

Our cross-sectional study is the first to examine the association between different traffic densities with cognitive performance in a large sample of Brazilian adults without dementia. We found that volunteers who live and work in regions with greater traffic density, and therefore more exposed to traffic air pollution, in the city of São Paulo did not present worse cognitive performance.

In general, studies showing the relationship between exposure to air pollutants and cognitive impairment included older individuals exposed to high concentrations of air pollutants. A study carried out in one of the cities with the lowest concentration of air pollutants in the world (Ontario, Canada), accompanying 2.1 million people for 13 years, found a higher incidence of dementia in volunteers with a mean age of 73.8 years exposed to higher concentrations of air pollutants (Chen et al. 2017). Jung et al. (2015), when assessing exposure to particulate matter in volunteers over 65 years of age, found that for the increase of an average of $4.34 \mu \mathrm{g} / \mathrm{m}^{3}$ of $\mathrm{PM}_{2.5}$, the risk of Alzheimer's disease rises 138\%. In the Nurses' Health study, which included 19,409 women between the ages of 70 and 81 years old, a positive association between prolonged exposure to $\mathrm{PM}_{2.5}$ and $\mathrm{PM}_{10}$ and cognitive decline was found in older women. Investigating the association between $\mathrm{PM}_{2.5}$ and mild cognitive impairment in women aged 68 to 79 years, Ranft et al. (2009) found a positive association between chronic exposure to $\mathrm{PM}_{2.5}$ and risk for Alzheimer's disease. Experimental studies have already described several pathophysiological pathways for this association. In a simplified way, regardless of the entry route of the air pollutants in the body, the pollutants triggered neuropathological lesions, such as the accumulation of $\beta$-amyloid protein, which culminated in neuronal death (CalderónGarcidueña et al. 2008 and Calderón-Garcidueñas et al. 2002).

However, some studies found different results from those mentioned above. Surprisingly, 
studies found lower risk of cognitive decline or dementia among those with greater exposure to air pollutants (Genc et al. 2012; Deguen et al. 2010; Forastiere et al. 2007). This negative association can be related to the socioeconomic status of individuals living or working in these regions, availability of urban infrastructure, and age of the population studied. Ribeiro et al. (2019), Habermann et al. (2014), and Forastiere et al. (2007) found in their studies a lesser effect of air pollutants of urban origin on the health of individuals with better socioeconomic status, even residents of regions with greater exposure to air pollutants. It is important to emphasize that the use and occupation of the urban space in the city of São Paulo began and intensified in the central region of the city. Although the central areas of the city have a greater density of traffic, and consequently higher levels of air pollution, the advantages of residing in the central region of São Paulo ends up concentrating residents with better socioeconomic status (Habermann et al. 2014). Another plausible explanation for our null findings may be related to the availability of urban infrastructure in the city of São Paulo and how much access to these public facilities can improve the quality of life of its inhabitants despite the exposure to high traffic density. Better quality public services, such as schools, hospitals, leisure options, work opportunities, broad public transportation coverage, and higher real estate value are concentrated in the central regions of the city, being available mainly to the population with higher socioeconomic status (Ribeiro et al. 2019; Morais et al. 2018). Similarly, the absence of adequate urban infrastructure and the presence of several social vulnerability factors in more peripheral areas explain why individuals with lower socioeconomic status, although not always exposed to higher concentrations of air pollutants, are the ones who suffer most from the deleterious health effects caused by pollutants (Denguen et al. 2010). In fact, when we adjusted our results for socioeconomic variables, previous significant associations became null. Another explanation for our results may be related to the low 
age of the volunteers in this study (mean age $=51$ years old), since studies that found a positive association between greater exposure to air pollutants and deficits in cognitive function examined older individuals. Possibly the deleterious effect of chronic exposure to pollution may be evident only later during the life course.

Although we could not measure individual exposure to different concentrations of air pollutants, an important feature of this study is the ability to estimate DWTD in individuals living in cities devoid of broad atmospheric monitoring, such as the city of São Paulo and several other cities in emerging countries. Since approximately $91 \%$ of the world population lives in places where air pollution exceeds the limits recommended by the World Health Organization and that $8 \%$ of all deaths in the world in 2016 were caused by exposure to air pollutants, alternative methodologies of air quality exposure assessment become extremely important in an environmental and public health context (WHO 2019). We also present here the first large study that assesses cognitive performance and exposure to air pollutants in a middle-income country. Another study strength is related to the fact that first cerebral lesions related to dementia can start decades before the cognitive symptoms (Sperling et al. 2014). Although cognitive impairment manifests in older individuals, the first cognitive alterations seem to start early. Therefore, studies that can identify subtle cognitive changes in younger individuals are important to identify early contributors to cognitive impairment. On the other hand, this study has some limitations. First, its cross-sectional design, which prevents it from presenting inferences about the effects of exposures to air pollutants and cognitive impairment. This study also did not take into account the time spent by volunteers in commuting, average age of the vehicle fleet, type of vehicle fleet (car, bus or truck), type of environment in which the volunteer is exposed to the pollutants (open or closed spaces), and climatic variables, such as wind direction. 


\section{Conclusion}

We did not find an association between exposure to higher traffic density and cognitive

performance. Follow-up of participants of this longitudinal study might be able to

elucidate if air pollutants are associated with cognitive decline and dementia.

\section{References}

Aquino EM, Barreto SM, Bensenor IM, Carvalho MS, Chor D, Duncan BB, Lotufo PA, Mill JG, Molina Mdel C, Mota EL, Passos VM, Schmidt MI, Szklo M. 2012. Brazilian Longitudinal Study of Adult Health (ELSA-Brasil): objectives and design. Am J Epidemiol 175(4):315-24.

Bennett DA, Schneider JA, Buchman AS, Barnes LL, Boyle PA and Wilson RS. 2012. Overview and findings from the rush Memory and Aging Project. Curr Alzheimer Res 9:646-63.

Bertolucci PH, Okamoto IH, Brucki SM, Siviero MO, Toniolo Neto J and Ramos LR. 2001. Applicability of the CERAD neuropsychological battery to Brazilian elderly. ArqNeuropsiquiatr 59:532-6.

Bertolucci PHF, Okamoto IH, Toniolo Neto J, Ramos LR, Brucki SMD. 1998. Desempenho da população brasileira na bateria neuropsicológica do Consortium to Establish a Registry for Alzheimer'sDisease (CERAD) [Performance of Brazilian population in neuropsychological battery of Consortium to Establish a Registry for Alzheimer'sdisease]. Rev Psiquiatr Clín (São Paulo) 25(2):80-3.

Calderón-Garcidueñas L1, Azzarelli B, Acuna H, Garcia R, Gambling TM, Osnaya N, Monroy S, DEL Tizapantzi MR, Carson JL, Villarreal-Calderon A, Rewcastle B. 2002. Air pollution and brain damage. Toxicologic Pathology 30(3): 373-389.

Calderón-Garcidueñas L, Mora-Tiscareño A, Ontiveros E, Gómez-Garza G, Barragán-Mejía G, Broadway J, Chapman S, Valencia-Salazar G, Jewells V, Maronpot RR, Henríquez-Roldán C, Pérez-Guillé B, TorresJardón R, Herrit L, Brooks D, Osnaya-Brizuela N, Monroy ME, González-Maciel A, Reynoso-Robles R, Villarreal-Calderon R, Solt AC, Engle RW. 2008. Air pollution, cognitive deficits and brain abnormalities: a pilot study with children and dogs," Brain and Cognition 68(2);117-127.

Calderón-Garcidueñas, Solt AC, Henríquez-Roldán C, Torres-Jardón R, Nuse B, Herritt L, VillarrealCalderón R, Osnaya N, Stone I, García R, Brooks DM, González-Maciel A, Reynoso-Robles R, DelgadoChávez R, Reed W. 2008. Long-term air pollution exposure is associated with neuroinflammation, an altered innate immune response, disruption of the blood-brain barrier, ultrafine particulate deposition, and accumulation of amyloid $\beta-42$ and $\alpha$-synuclein in children and young adults. Toxicologic Pathology 36(2):289-310.

Chaimowicz, F. 1997. A saúde dos idosos brasileiros às vésperas do século XXI: problemas, projeções e alternativas. Rev Saúde Publica 31(2): 184-200.

Chen H, Kwong JC, Copes R, Hystad P, van Donkelaar A, Tu K, Brook JR, Goldberg MS, Martin RV, Murray BJ, Wilton AS, Kopp A, Burnett RT. 2017. Exposure to ambient air pollution and the incidence of dementia: A population-based cohortstudy. Environ Int 108:271-277.

Clifford A, Lang L, Chen R, Anstey KJ, Seaton A. 2015. Exposure to air pollution and cognitive functioning across the life course - A systematic literature review. Int J Epidemiol 44(1):68-75.

Deguen S and Zmirou-Navier D. 2010. Social inequalities resulting from health risks related to ambient air quality--A European review. Eur J Public Health 20(1):27-35. 
Duron, E.; Hanon, O. 2008. Hypertension, cognitive decline and dementia. Arch of Cardiovasc Diseases 101: 181-189.

Ferri PF. 2012. Population ageing in Latin America: dementia and related disorders". Ver Bras Psiquiatr 34:371-374.

Forastiere F, Stafoggia M, Tasco C, Picciotto S, Agabiti N, Cesaroni G, PerucciCA. 2007. Socioeconomic status, particulate air pollution, and daily mortality: differential exposure or differential susceptibility. Am J Ind Med 50:208-16.

Forlenza OV1, Diniz BS, Stella F, Teixeira AL, Gattaz WF. 2013. Mild Cognitive Impairment (part 1): clinical characteristics and predictors of dementia. Rev Bras Psiquiatr 35: 178-185.

Greenlief CL, Margolis RB and Erker GJ. 1985. Application of the Trail Making Test in differentiating neuropsychological impairment of elderly persons. Percept Mot Skills. 61:1283-9.

Genc S, Zadeoglulari Z, Fuss SH, Genc K. 2012. The adverse effects of air pollution on the nervous system. J Toxicol 2012:782462.

Habermann M, Medeiros APP, Gouveia N. 2011. Motor vehicle traffic as an air pollution exposure assessment method in big cities. Rev Bras Epidemiol 14(1):120-30.

Habermann M, Souza M, Prado R, Gouveia N. 2014. Inequalities and exposure to traffic-related air pollution in the city of São Paulo, Brazil. Cad. SaúdePública, Rio de Janeiro 30(1):119-125.

Jung, C.R., Lin, Y.T., Hwang, B.F. 2015. Ozone, particulate matter, and newly diagnosed Alzheimer's disease: a population-based cohort study in Taiwan. J. Alzheimers Dis 44:573-584.

Lewis G, Pelosi AJ, Araya R and Dunn G. 1992. Measuring psychiatric disorder in the community: a standardized assessment for use by lay interviewers. Psychol Med. 22:465-86.

Mendes PM, Nobre AA, Griep RH, Joanna MNG, Leidjaira LJ, Barreto SM, Pereira A, Chor A. 2018. Asociación entre la discriminación racial percibida y la hipertensión: hallazgos del estudio ELSA-Brasil. Cad. Saúde Pública 34(2):e00050317.

Morais GAS, Sobreira DB, Lima JE. 2018. Padrão e Determinantes Da Infraestrutura Urbana das Microrregiões Brasileiras. Geosul, Florianópolis 33(66):262-291.

Morris JC, Heyman A, Mohs RC, Hughes JP, van Belle G, Fillenbaum G, Mellits ED and Clark C. 1989. The Consortium to Establish a Registry for Alzheimer's Disease (CERAD). Part I. Clinical and neuropsychological assessment of Alzheimer's disease. Neurology 39:1159-65.

Nitrini R, Caramelli P, Herrera Júnior E, Porto CS, Charchat-Fichman H, Carthery MT, Takada LT, Lima EP. 2004. Performance of illiterate and literate nondemented elderly subjects in two tests of long-term memory. J IntNeuropsychol Soc 10(4):634-8.

Nitrini R, Caramelli P, Herrera E Jr, Bahia VS, Caixeta LF, Radanovic M, Anghinah R, Charchat-Fichman H, Porto CS, Carthery MT, Hartmann AP, Huang N, Smid J, Lima EP, Takada LT, Takahashi DY. 2004. Incidence of dementia in a community-dwelling Brazilian population. Alzheimer Dis Assoc Disord $18(4): 241-246$.

Nitrini R, Bottino CM, Albala C, Custodio Capuñay NS, Ketzoian C, Llibre Rodriguez JJ, Maestre GE, Ramos-Cerqueira AT, CaramelliP. 2009. Prevalence of dementia in Latin America: a collaborative study of population-based cohorts". InternlPsychoger 21(4):622-630.

NIH (National Institute of Health). 2013. The dementias: hope through research.

Oudin A1, Forsberg B, Adolfsson AN, Lind N, Modig L, Nordin M, Nordin S, Adolfsson R, Nilsson LG. 2016. Traffic-related air pollution and dementia incidence in northern Sweden: a longitudinal study. Environ. Health Perspect 124:306-312. 
Passos VM, Caramelli P, Bensenor I, Giatti L and Barreto SM. 2014. Methods of cognitive function investigation in the Longitudinal Study on Adult Health (ELSA-Brasil). São Paulo Med J 132:170-7.

Parra MA, Baez S, Allegri R, Nitrini R, Lopera F, Slachevsky A, Custodio N, Lira D, Piguet O, Kumfor F, Huepe D, Cogram P, Bak T, Manes F, Ibanez A. 2018. Dementia in Latin America: Assessing the present and envisioning the future. Neurology 30;90(5):222-231.

Pearson RL, Wachtel H, Ebi KL. 2000. Distance-weighted traffic density in proximity to a home is a risk factor for leukemia and other childhood cancers. Air Waste Manag Assoc 50: 175-80.

Prince M, Bryce R, Albanese E, Wimo A, Ribeiro W, FerriCP. 2013. The global prevalence of dementia: a systematic review and metaanalysis". Alzheimer \& Dementia 9: 63-75.

Ranft, U., Schikowski, T., Sugiri, D., Krutmann, J., Kramer, U. 2009. Long-term exposure to traffic-related particulate matter impairs cognitive function in the elderly. Environ. Res 109:1004-1011.

Rawlings AM, Sharrett AR, Schneider AL, Coresh J, Albert M, Couper D, Griswold M, Gottesman RF, Wagenknecht LE, Windham BG and Selvin E. 2014. Diabetes in midlife and cognitive change over 20 years: a cohort study. Ann Intern Med 161:785-93.

Ribeiro AG, Baquero OS, Almeida SL, Freitas CU, Cardoso MRA, Nardocci AC. 2019. Influence of vehicular traffic density on hospital admissions due to respiratory tract cancer in the city of São Paulo, Brazil. Reports in Public Health 35(1), e00128518.

Schmidt MI, Griep RH, Passos VM, Luft VC, Goulart AC, Menezes GM. 2013. Strategies and development of quality assurance and control in the ELSA-Brasil. Rev Saude Publica 47 Suppl 2:105-12.

Schmidt MI, Duncan BB, Mill JG, Lotufo PA, Chor D, Barreto SM, Aquino EM, Passos VM, Matos SM, Molina MD, Carvalho MS and Bensenor IM. 2014. Cohort Profile: Longitudinal Study of Adult Health (ELSA-Brasil). Int J Epidemiol 44(1):68-75.

Shao Z, Janse E, Visser K and Meyer AS. 2014. What do verbal fluency tasks measure? Predictors of verbal fluency performance in older adults. Frontiers in Psychology 5:772.

Suemoto CK, Baena CP, Mill JG, Santos IS, Lotufo PA, Benseñor I. 2018. Orthostatic Hypotension and Cognitive Function: Cross-sectional Results From the ELSA-Brasil Study. J Gerontol A BiolSci Med Sci. $00: 1-8$.

Sperling R, Mormino E and Johnson K. 2014. The evolution of preclinical Alzheimer's disease: Implications for prevention trials. Neuron 84(3): 608-622.

Tuokko H, Frerichs R, Graham J, Rockwood K, Kristjansson B, Fisk J, Bergman H, Kozma A, McDowell I. 2003. Five-year follow-up of cognitive impairment with no dementia. Arch Neurol 60(4):577-82.

WAR (World Alzheimer Report). 2014. World Alzheimer’s Disease International.

WAR (World Alzheimer Report). 2015. World Alzheimer’s Disease International.

WHO (World Health Organization). 2006. Air quality guidelines. Global update 2005. Particulate matter, ozone, nitrogen dioxide and sulfur dioxide.

WHO (World Health Organization). 2012. Dementia: a public health priority.

WHO (World Health Organization). 2018. Ambient air pollution - a major threat to health and climate. 


\section{SUPPLEMENTARY MATERIAL}

Supplementary Table 1. Characteristics of the study population $(n=3,050)$

\begin{tabular}{lc}
\hline Variable & Mean (SD) or \% \\
\hline Immediate word recall, mean (SD) & $20(3.8)$ \\
Delayed word recall, mean (SD) & $6.6(1.9)$ \\
Word recognition, mean (SD) & $9.5(0.8)$ \\
Semantic verbal fluency, mean (SD) & $18.5(5.0)$ \\
Phonemic verbal fluency, mean (SD) & $12.5(4.4)$ \\
Trail making test (seconds), mean (SD) & $119.5(84.9)$ \\
Traffic Density Weighted by Distance, mean (SD) & $544.5(879.4)$ \\
Age (years), mean (SD) & $52.1(9.2)$ \\
Male, \% & 43.5 \\
Marital, \% & \\
Married & 65.51 \\
Separate & 14.9 \\
Single & 12.9 \\
Widower & 3.8 \\
Other & 2.8 \\
Race, \% & \\
Black & 11.5 \\
Brown & 18.3 \\
White & 63.6 \\
Asian & 5.7 \\
Indigenous & 0.9 \\
Education, \% & \\
Less than Elementary school & \\
Less than middle school & 5.0 \\
Complete high school & 6.2 \\
University degree & 34.6 \\
Diabetes, \% & 54.1 \\
Hypertension, \% & 19.7 \\
LDL-cholesterol (mg/dl), mean (SD) & 31.6 \\
HDL-cholesterol (mg/dl), mean (SD) & $129.7(33.8)$ \\
Alcohol use, $\%$ & $57.0(14.3)$ \\
Never & \\
Former & 10.4 \\
Current & 17.6 \\
Smoking, \% & 72.0 \\
Never & \\
Former & 53.4 \\
Current & 30.4 \\
Physical activity, \% & 16.0 \\
No & \\
Yes <150 min/wk & 76.0 \\
Yes, $\geq 150$ min/wk & 14.1 \\
Body mass index (Kg/m ${ }^{2}$ ), mean (SD) & 9.9 \\
Depression, \% & $27.1(4.9)$ \\
Cardiovascular disease, $\mathrm{n}(\%)$ & 3.8 \\
\hline & 2.7 \\
\hline
\end{tabular}


Supplementary Table 2. Association of cognitive performance with Traffic Density Weighted by Distance (DWTD) near participant's workplace ( $n=3,050)$

\begin{tabular}{|c|c|c|c|c|c|c|c|c|c|}
\hline \multirow[b]{2}{*}{ z-Score } & \multirow[b]{2}{*}{ Tercis } & \multirow{2}{*}{$\begin{array}{l}\text { Crude } \\
\beta(95 \% \mathrm{CI})\end{array}$} & \multirow[b]{2}{*}{$p$} & Model 1 & \multirow[b]{2}{*}{$p$} & Model 2 & \multirow[b]{2}{*}{$P$} & Model 3 & \multirow[b]{2}{*}{$p$} \\
\hline & & & & $\beta(95 \% C I)$ & & $\beta(95 \% C I)$ & & $\beta(95 \% C I)$ & \\
\hline \multirow{2}{*}{$\begin{array}{l}\text { Composite } \\
\text { global }\end{array}$} & 2nd & $-0.184(-0.262 ;-0.106)$ & \multirow[t]{2}{*}{$<0.0001$} & $-0.254(-0.329 ;-0.178)$ & \multirow[t]{2}{*}{$<0.0001$} & $-0.058(-0.123 ; 0.006)$ & \multirow[t]{2}{*}{0.19} & $-0.053(-0.118 ; 0.011)$ & \multirow[t]{2}{*}{0.25} \\
\hline & $3 \mathrm{rd}$ & $-0.065(-0.148 ; 0.016)$ & & $-0.085(-0.164 ;-0.005)$ & & $-0.011(-0.078 ; 0.056)$ & & $-0.011(-0.079 ; 0.055)$ & \\
\hline \multirow[t]{2}{*}{ Memory } & 2nd & $-0.063(0 .-143 ; 0.017)$ & \multirow[t]{2}{*}{0.25} & $-0.142(-0.220 ;-0.065)$ & \multirow[t]{2}{*}{0.001} & $-0.025(-0.099 ; 0.049)$ & \multirow[t]{2}{*}{0.58} & $-0.023(-0.097 ; 0.051)$ & \multirow[t]{2}{*}{0.61} \\
\hline & $3 \mathrm{rd}$ & $-0.001(-0.087 ; 0.083)$ & & $-0.027(-0.109 ; 0.053)$ & & $0.017(-0.059 ; 0.095)$ & & $0.018(-0.058 ; 0.096)$ & \\
\hline \multirow{2}{*}{$\begin{array}{l}\text { Verbal } \\
\text { fluency }\end{array}$} & 2nd & $-0.165(0.247 ;-0.083)$ & \multirow[t]{2}{*}{0.0004} & $-0.193(-0.255 ;-0.111)$ & \multirow[t]{2}{*}{$<0.0001$} & $-0.012(-0.087 ; 0.062)$ & \multirow[t]{2}{*}{0.91} & $-0.007(-0.082 ; 0.066)$ & \multirow[t]{2}{*}{0.97} \\
\hline & $3 \mathrm{rd}$ & $-0.188(-0.137 ; 0.035)$ & & $-0.060(-0.146 ; 0.026)$ & & $0.003(-0.074 ; 0.081)$ & & $0.001(-0.076 ; 0.078)$ & \\
\hline \multirow{2}{*}{$\begin{array}{l}\text { Trail } \\
\text { making }\end{array}$} & 2nd & $-0.321(-0.399 ;-0.243)$ & \multirow[t]{2}{*}{$<0.0001$} & $-0.365(-0.442 ;-0.289)$ & \multirow[t]{2}{*}{$<0.0001$} & $-0.156(-0.220 ;-0.093)$ & \multirow[t]{2}{*}{$<0.0001$} & $-0.151(-0.214 ;-0.087)$ & \multirow[t]{2}{*}{$<0.0001$} \\
\hline & 3rd & $-0.178(-0.260 ;-0.096)$ & & $-0.177(-0.258 ;-0.096)$ & & $-0.096(-0.164 ;-0.030)$ & & $-0.096(-0.162 ;-0.030)$ & \\
\hline
\end{tabular}

Model 1: Linear regression model adjusted for age and sex.

Model 2: Linear regression model adjusted for age, sex, race, and education.

Model 3: Linear regression model adjusted for age, sex, race, education, LDL-cholesterol, HDL-cholesterol, hypertension, diabetes, cardiovascular disease, smoking,

body mass index, alcohol use, physical activity, depression, and number of years in the current workplace.

Reference: $1^{\text {st }}$ tertile of DWTD 
Supplementary Table 3. Association of cognitive performance with Traffic Density Weighted by Distance (DWTD) near participant's residence (n $=3050)$

\begin{tabular}{|c|c|c|c|c|c|c|c|c|c|}
\hline \multirow[b]{2}{*}{ z-Score } & \multirow[b]{2}{*}{ Tercis } & Crude & \multirow[b]{2}{*}{$p$} & Model 1 & \multirow[b]{2}{*}{$p$} & Model 2 & \multirow[b]{2}{*}{$p$} & Model 3 & \multirow[b]{2}{*}{$p$} \\
\hline & & $\beta(95 \% \mathrm{CI})$ & & $\beta(95 \% C I)$ & & $\beta(95 \% C I)$ & & $\beta(95 \% C I)$ & \\
\hline \multirow{2}{*}{$\begin{array}{c}\text { Composite } \\
\text { global }\end{array}$} & 2nd & $0.166(0.085 ; 0.247)$ & \multirow[t]{2}{*}{$<0.0001$} & $0.177(0.099 ; 0.254)$ & \multirow[t]{2}{*}{$<0.0001$} & $0.037(-0.029 ; 0.104)$ & \multirow[t]{2}{*}{0.01} & $0.033(-0.033 ; 0.100)$ & \multirow[t]{2}{*}{0.01} \\
\hline & $3 \mathrm{rd}$ & $0.290(0.209 ; 0.372)$ & & $0.338(0.259 ; 0.417)$ & & $0.103(0.035 ; 0.172)$ & & $0.097(-0.029 ; 0.166)$ & \\
\hline \multirow{2}{*}{ Memory } & 2nd & $0.084(0.001 ; 0.167)$ & \multirow{2}{*}{0.0002} & $0.091(0.012 ; 0.171)$ & \multirow[t]{2}{*}{$<0.0001$} & $0.007(-0.069 ; 0.083)$ & \multirow[t]{2}{*}{0.06} & $0.004(-0.072 ; 0.080)$ & \multirow[t]{2}{*}{0.05} \\
\hline & $3 \mathrm{rd}$ & $0.179(0.095 ; 0.263)$ & & $0.225(0.144 ; 0.306)$ & & $0.083(0.004 ; 0.161)$ & & $0.078(-0.001 ; 0.156)$ & \\
\hline \multirow{2}{*}{$\begin{array}{l}\text { Verbal } \\
\text { fluency }\end{array}$} & 2nd & $0.198(0.113 ; 0.282)$ & \multirow[t]{2}{*}{$<0.0001$} & $0.202(0.118 ; 0.286)$ & \multirow[t]{2}{*}{$<0.0001$} & $0.070(-0.006 ; 0.146)$ & \multirow[t]{2}{*}{0.05} & $0.068(-0.008 ; 0.146)$ & \multirow[t]{2}{*}{0.06} \\
\hline & $3 \mathrm{rd}$ & $0.288(0.203 ; 0.373)$ & & $0.308(0.222 ; 0.393)$ & & $0.092(0.013 ; 0.170)$ & & $0.089(0.010 ; 0.168)$ & \\
\hline \multirow[t]{2}{*}{ Trail making } & $2 \mathrm{nd}$ & $0.140(0.060 ; 0.221)$ & \multirow[t]{2}{*}{$<0.0001$} & $0.158(0.079 ; 0.238)$ & \multirow[t]{2}{*}{$<0.0001$} & $0.016(-0.049 ; 0.081)$ & \multirow[t]{2}{*}{0.12} & $0.012(-0.052 ; 0.077)$ & \multirow[t]{2}{*}{0.19} \\
\hline & $3 \mathrm{rd}$ & $0.265(0.183 ; 0.346)$ & & $0.315(0.234 ; 0.396)$ & & $0.066(-0.001 ; 0.134)$ & & $-0.058(-0.008 ; 0.125)$ & \\
\hline
\end{tabular}

Model 1: Linear regression model adjusted for age and sex.

Model 2: Linear regression model adjusted for age, sex, race, and education.

Model 3: Linear regression model adjusted for age, sex, race, education, LDL-cholesterol, HDL-cholesterol, hypertension, diabetes, cardiovascular disease, smoking, body mass index, alcohol use, physical activity, depression, and number of years living in the current residency.

Reference: $1^{\text {st }}$ tertile of DWTD 


\section{CONSIDERAÇÕES FINAIS}

Aqui, apresentamos o primeiro estudo a examinar a associação entre diferentes densidades de tráfego e desempenho cognitivo em uma grande amostra de adultos brasileiros sem demência. Constatamos que os voluntários que vivem e trabalham em regiões com maior densidade de tráfego e, portanto, estão mais expostos à poluição do ar originadas a partir do trânsito veícular, na cidade de São Paulo não apresentaram necessáriamente pior desempenho cognitivo. Esse achado, está relacionado a forma como a cidade de São Paulo se organiza, tendo pessoas com maior nível de escolaridade e consequentemente com maior renda vivendo em regiões com maior DTPD e pessoas com menor escolaridade e com menor renda vivendo em regiões com menor DTPD. No entanto, a exposição aos poluentes do ar e os danos ocasionados por essa exposição não é igualmente proporcional a escolaridade e renda, merecendo assim, ser melhor estudada. Outros elementos que aqui tratamos como limitações por não estarem disponíveis neste momento (tempo gasto pelos voluntários no deslocamento, idade média da frota de veículos da cidade de São Paulo, tipo de frota de veículos (carro, ônibus ou caminhão), tipo de ambiente em que o voluntário é exposto aos poluentes (abertos ou espaços fechados) e variáveis climáticas, como a direção do vento), também merecem ser inseridos em um novo desenho de estudo e explorados para evidenciar com mais força a relação entre a exposição aos poluentes do ar e comprometimento cognitivo e demência.

No entanto, conseguimos aqui calcular a DTPD individual para cada membro deste estudo agregando assim, valor a nossa análise e uma nova perspectiva de mensurar a exposição ambiental aos poluentes do ar em indivíduos que vivem em cidades desprovidas de amplo monitoramento atmosférico, como é o caso da cidade de São Paulo e de tantas outras cidades espalhadas pelo Brasil e pelo mundo. Seguindo o mesmo caminho, embora o comprometimento cognitivo se manifeste em indivíduos mais velhos, as primeiras alterações cognitivas parecem começar precocemente. Neste sentido, estudos como o aqui apresentado tem potencial para identificar alterações cognitivas sutis em indivíduos mais jovens e assim, estimular o desenvolvimento de tratamentos médicos bem como o desenvolvito de politicas publicas voltadas a mitigar a emissão de poluentes. O que estimula o desenvolvimento de novos estudos, com dados mais recentes e completos para evidenciar de forma mais consistente a relação entre a exposição aos poluentes atmosféricos de origem veicular e comprometimento cognito/demência. 


\section{REFERÊNCIAS BIBLIOGRÁFICAS}

Adam M. Brickman, et al. Category and letter verbal fluency across the adult lifespan:relationship to EEG theta power. Archives of Clinical Neuropsychology.2005;20:561-573.

Aquino EM, Barreto SM, Bensenor IM, et al. Brazilian Longitudinal Study of Adult Health (ELSA-Brasil): objectives and design. Am J Epidemiol. 2012; 175(4):315-24.

Bertolucci PHF, Okamoto IH, Toniolo Neto J, Ramos LR, Brucki SMD. Desempenho da população brasileira na bateria neuropsicológica do Consortium to Establish a Registry for Alzheimer's Disease (CERAD) [Performance of Brazilian population in neuropsychological battery of Consortium to Establish a Registry for Alzheimer's disease]. Rev Psiquiatr Clín (São Paulo). 1998;25(2):80-3.

Burlá, C. et al. "Panorama prospectivo das demências no Brasil: um enfoque demográfico". Cien Saude Colet 18, no 10 (2013): 2949-56.

Brucki SMD, Malheiros SMF, Okamoto IH, Bertolucci PHF. Dados normativos para o teste de fluência verbal categoria animais em nosso meio. ArqNeuropsiquiat 1997;55(1):56-61.

Block ML, Calderón-Garcidueñas L. "Air pollution: ^ mechanisms of neuroinflammation and CNS disease," Trends in Neurosciences. 2009. vol. 32, no. 9, pp. 506-516.

Cacciottolo M, Wang X, Driscoll I, Woodward N, Saffari A, Reyes J, Serre ML, Vizuete W, Sioutas C, Morgan TE, Gatz M, Chui HC, Shumaker SA, Resnick SM, Espeland MA, Finch CE, Chen JC. Particulate air pollutants, APOE alleles and their contributions to cognitive impairment in older women and to amyloidogenesis in experimental models. Transl. Psychiatry 2017. 7, e1022.

Calderón-Garcidueñas L1, Mora-Tiscareño A, Ontiveros E, Gómez-Garza G, Barragán-Mejía G, Broadway J, Chapman S, Valencia-Salazar G, Jewells V, Maronpot RR, Henríquez-Roldán C, Pérez-Guillé B, Torres-Jardón R, Herrit L, Brooks D, Osnaya-Brizuela N, Monroy ME, González-Maciel A, Reynoso-Robles R, Villarreal-Calderon R, Solt AC, Engle RW. "Air pollution, cognitive deficits and brain abnormalities: a pilot study with children and dogs,” Brain and Cognition, vol. 68, no. 2, pp. 117-127, 2008.

Calderón-Garcidueñas L, Reed W, Maronpot RR, Henríquez-Roldán C, Delgado-Chavez R, Calderón-Garcidueñas A, Dragustinovis I, Franco-Lira M, Aragón-Flores M, Solt AC, Altenburg M, Torres-Jardón R, Swenberg JA. "Brain inflammation and Alzheimer's-like pathology in individuals exposed to severe air pollution," Toxicologic Pathology 2004. vol. 32, no. 6, pp. 650-658.

Calderón-Garcidueñas L, Azzarelli B, Acuna H, Garcia R, Gambling TM, Osnaya N, Monroy S, DEL Tizapantzi MR, Carson JL, Villarreal-Calderon A, Rewcastle B. “Air pollution and brain damage”. Toxicologic Pathology, 2002. 30(3): 373-389.

Calderón-Garcidueñas L, Solt AC, Henríquez-Roldán C, Torres-Jardón R, Nuse B, Herritt L, Villarreal-Calderón R, Osnaya N, Stone I, García R, Brooks DM, González-Maciel A, Reynoso-Robles R, Delgado-Chávez R, Reed W. "Long-term Air Pollution Exposure Is Associated with Neuroinflammation, an Altered Innate Immune Response, Disruption of the Blood-Brain Barrier, Ultrafine Particulate Deposition, and Accumulation of Amyloid $\beta-42$ and $\alpha$ Synuclein in Children and Young Adults". Toxicologic Pathology, 2008. 36: 289-310.

Caracciolo B, Palmer K, Monastero R, Winblad B, Bäckman L, Fratiglioni L. "Occurrence of cognitive impairment and dementia in the community: a 9-year-long prospective study". Neurology 2008; 70: 1778-1785.

Carey IM, Atkinson RW, Kent AJ, van Staa T, Cook DG, Anderson HR. "Mortality Associations with Long-Term Exposure to Outdoor Air Pollution in a National English Cohort". Am J Res Crit Care Med, 2013. 187 (11): 12261233.

CETESB - Companhia Ambiental do Estado de São Paulo. Qualidade do ar no Estado de São Paulo 2012. São Paulo: CETESB, 2013. 
Chaimowicz, F. “A saúde dos idosos brasileiros às vésperas do século XXI: problemas, projeções e alternativas”. Rev Saú Pub, 1997. Vol 31. 2: 184-200.

Chang, K.H., Chang, M.Y., Muo, C.H., Wu, T.N., Chen, C.Y., Kao, C.H., Increased risk of dementia in patients exposed to nitrogen dioxide and carbon monoxide: a population-based retrospective cohort study. PLoS One 9, 2014. E103078.

Consenza RM, Avaliação neuropsicológica do Idoso. In: Tavares A. Compêndio de neuropriquiatria geriátrica. 1a ed. Rio de Janeiro:Guanabara Koogan; 2005.P. 157-173.

Chen H, Kwonga JC, Copesa R, Hystad P, Donkelaar AV, Tub K, Brookc JR, Goldberg MS, Martinf RV, Murray B, Wilton AS, Kopp A, Burnett RT. Exposure to ambient air pollution and the incidence of dementia: A populationbased cohort study. Environment International. 2017. 108: 271-277

Daniels MJ, Dominici F, Samet JM, Ze ger SL. Estimating particulate matter mortality dose-response curves and threshold levels: an analysis of daily time-series for the 20 largest US cities. Am J Epidemiol 2000; 152: $397-406$.

Darrow LA, Klein M, Flanders WD, Mulholland JA, Tolbert PE, Strickland MJ. "Air pollution and acute respiratory infections among children 0-4 years of age: an 18-years' time-series study”. AmJEp,2014. 180(10): 968-977.

Fagundes SD, Silva MT, Thees MF, Pereira MG. "Prevalence of dementia among elderly Brazilians: a systematic review". São Paulo Med J, 2011; 129(1): 46-50.

Faustini A, Stafoggia M, Berti G, Bisanti L, Chiusolo M, Cernigliaro A, Mallone S, Primerano R, Scarnato C, Simonato L, Vigotti MA, Forastiere F; EpiAir Collaborative Group. "The relationship between ambient particulate matter and respiratory mortality: a multi-city study in Italy”. Eur Respir J, 2011. 38: 538-547.

Genc S, Zadeoglulari Z, Fuss SH, Genc K. "The adverse effects of air pollution on the nervous system". $J$ of Toxicology, 2012. 23p.

Jung, C.R., Lin, Y.T., Hwang, B.F. Ozone, particulate matter, and newly diagnosed Alzheimer's disease: a population-based cohort study in Taiwan. J. Alzheimers Dis. 2015. 44, 573-584.

Künzli N1, Jerrett M, Garcia-Esteban R, Basagaña X, Beckermann B, Gilliland F, Medina M, Peters J, Hodis HN, Mack WJ. Ambient air pollution and the progression of atherosclerosis in adults.PLoS One. 2010 Feb 8; 5(2):e9096.

Landrigan PJ, Fuller R, Acosta NJR, Adeyi O, Arnold R, Basu N, Baldé AB, Bertollini R, Reilly TB, Boufford JI, Breysse PN, Chiles C, Mahidol C, Coll-Seck AM, Cropper ML, Fobil J, Fuster V, Greenstone M, Haines A, Hanrahan D, Hunter D, Khare M, Krupnick A, Lanphear B, Lohani B, Martin K, Mathiasen KV, McTeer MA, Murray CJL, Ndahimananjara JD, Perera F, Potočnik J, Preker AS, Ramesh J, Rockström J, Salinas C, Samson LD, Sandilya K, Sly PD, Smith KR, Steiner A, Stewart RB, Suk WA, Schayck OCP, Yadama GN, Yumkella K, Zhong M. The Lancet Commission on pollution and health. Sci Total Environ. 2019; 10;650(Pt 2):2389-2394.

Lezak, MD. Neuropsychological Assessment. 3rded. New York: Oxford University Press, 1995.

Li N, Hao M, Phalen RF, Hinds WC, Nel AE. Particulate air pollutants and asthma. A paradigm for the role of oxidative stress in PM-induced adverse health effects. Clin Immunol 2003; 109: 250-265.

Machado FP, Avaliação neuropsicológica do Idoso. In: Tavares A. Compêndio de neuropsiquiatria geriátrica. 2a ed. Rio de Janeiro: Guanabara Koogan; 2005. P. 157-173.

McMichael AJ, Woodward A, Muir C. Climate change and the health of nations: famines, fevers, and the fate of populations. Oxford: Oxford University Press, 2017.

Nitrini R, Caramelli P, Herrera Júnior E, et al Performance of illiterate and literate nondemented elderly subjects in two tests of long-term memory. J Int Neuropsychol Soc. 2004;10(4):634-8.

Nitrini R, Bottino CM, Albala C, Custodio Capuñay NS, Ketzoian C, Llibre Rodriguez JJ, Maestre GE, RamosCerqueira AT, Caramelli P. "Prevalence of dementia in Latin America: a collaborative study of population-based cohorts". Internl Psychoger, 2009. 21: 4, 622-630. 
Numan, M.; Brown, J.; Michou, L. "Impact of air pollutants on oxidative stress in common autophagy-mediated aging diseases". Int J Environ Res Public Health, 2015. 12: 2289-2305.

Nunomura A, Castellani RJ, Zhu X, Moreira PI, Perry G, Smith MA. "Involvement of oxidative stress in Alzheimer disease". J Neuropathol, 2006. 65(7): 631-641.

OECD - Organization for Economic Co-operation and Development. OECD Environmental Outlook to 2050: The Consequences of Inaction. OECD: 2011.

Oudin A, Forsberg B, Adolfsson AN, Lind N, Modig L, Nordin M, Nordin S, Adolfsson R, Nilsson LG. "TrafficRelated Air Pollution and Dementia Incidence in Northern Sweden: A Longitudinal Study”. Environmental Health Persp 2015.

Oudin, A., Forsberg, B., Adolfsson, A.N., Lind, N., Modig, L., Nordin, M., et al. Traffic-related air pollution and dementia incidence in northern Sweden: a longitudinal study. Environ. Health Perspect. 2016. 124, 306-312.

OLMO NRS. Poluição Atmosférica e Exposição Humana: a evolução científica epidemiológica e sua correlação com o ordenamento jurídico. 2010.Tese de Doutorado. Faculdade de Medicina da Universidade de São Paulo - Programa de Pós-Graduação em Ciências. São Paulo, Brasil.

Ostro B. Outdoor air pollution. In; World Health Organization. Environmental Burden of Diseases Series 2004, N.5.

Passos VM, Caramelli P, Benseñor I, Giatti L, Barreto SM. "Methods of cognitive function investigation in the Longitudinal Study on Adult Health (ELSA-Brasil)" Sao Paulo Med J. 2014. 132(3):170-7.

Pope CA; Thun MJ; Namboodiri MM; Dockery DW; Evans JS; Speizer FE; Heath CW jr. Particulate air pollution as a predictor of mortality in a prospective study of US adults. American Journal of Respiratory and Critical Care Medicine. 19985; 151(3):669-74.

Power MC, Weisskopf MG, Alexeeff SE, Coull BA, Spiro A 3rd, Schwartz J. "Traffic-Related Air Pollution and Cognitive Function in a Cohort of Older Men" Environ Health Perspect 119:682-687 (2011). doi:10.1289/ehp.1002767.

Prince M, Bryce R, Albanese E, Wimo A, Ribeiro W, Ferri CP. "The global prevalence of dementia: a systematic review and meta-analysis". Alzheimer \& Dementia, 2013. 9: 63-75.

Pruss-Ustun A, Bonjour S, Covalan C. The impact of the environment on health by country: a meta-synthesis. Environ Health. 2008; 7(7):200.

Ranft U, Schikowski T, Sugiri D, Krutmann J, Kramer U. Long-term exposure to traffic-related particulate matter impairs cognitive function in the elderly. Environ. Res. 2009. 109, 1004-1011.

Rockström J, Steffen W, Noone K. A safe operating space for humanity. Nature 2009; 461: 472-75.

Tonne C, Elbaz A, Beevers S, Singh-Manoux A. "Traffic-related Air Pollution in Relation to Cognitive Function in Older Adults". Epidemiology 2014;25: 674-681.

Tuokko H, Frerichs R, Graham J. Five-year follow-up of cognitive impairment with no dementia. Arch Neurol. 2003;60(4):577-82.

Tuppo E; ARIAS H. "The role of inflammation in Alzheimer's disease". The international $J$ of Biochem \& Cell Biology, 2005. 37: 289-305.

Vormittag, EMPA; Rodrigues, CG; Miranda, MJ; Cavalcante, JA; Costa, RR; Camargo, CA; Saldiva, PHN. Avaliação do impacto da poluição atmosférica no estado de São Paulo sob a visão da saúde. Instituto Saúde e Sustentabilidade. 2013;1(1):1-75.

Wellenius GA, Boyle LD, Coull BA, Milberg WP, Gryparis A, Schwartz J. Residential proximity to nearest major roadway and cognitive function in community-dwelling seniors: results from the MOBILIZE Boston study. J. Am. 
Geriatr. Soc. 2012. 60, 2075-2080.

Whitmee S, Haines A, Beyrer C, et al. Safeguarding human health in the Anthropocene epoch: report of The Rockefeller Foundation-Lancet Commission on planetary health. Lancet 2015; 386:1973-2028.

Wilker, E.H., Preis, S.R., Beiser, A.S., Wolf, P.A., Au, R., Kloog, I., et al. Long-term exposure to fine particulate matter, residential proximity to major roads and measures of brain structure. Stroke. 2015. 46, 1161-1166.

WorldAltheimer'sDiseaseIntemational. World Alzheimer Report 2009. Londres, 2009. 96p.

WorldAlzheimer'sDiseaseInternational.WorldAlzheimer Report2010. Londres, 2011. 56p.

WorldAlzheimer'sDiseaseInternational. World Alzheimer Report 2014. Londres, 2014. 104p.

World Health Organization. Air quality guidelines. Global update 2005. Particulate matter, ozone, nitrogen dioxide and sulfur dioxide. Copenhagen: World Health Organization; 2006.

World Health Organization. Dementia: a public health priority. United Kingdom, 2012. 112p.

Wu et al. "Association between air pollutants and dementia risk in the elderly". Alzh \& Dementia 2015; 220-8. 


\section{APÊNDICES}

\section{APÊNDICE A: PARECER CEP DA PESQUISA-BASE}

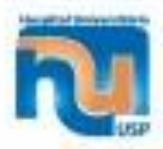

Sfo Paulo, 23 de outulero de 2018

$$
r^{\text {enes }}, 5^{\text {nis }} \text {. }
$$

Dra Clawila Kinre Siremoto

Pespisiodaria) respowsived

Faculdade de Medicina

UNIVERSIDADE DE SAO PAULO

REFERENTE: Projeto de Pesquisa "Poluigalo atmosférica como Anor de risco para o declinio cegnitivo no Estudo Longitudinal da Saude do Adulto - ELSA-Brasir

Equipe de Pesquisa: Douglas Rene Rocha Silva, Nelson da Cruz Gourveia, Isabela Jukith Martins Bensetiot, Paulo Andrade Lotufo

CAAE: 96808518.9.0000.0076

Registro CEP-HU/USP: 1741/18

Prezado(a) Senhor(a)

O Comitêt de Etica em Pesquisa do Hocpital Universithrio da Universidade de Sko Pasalo, em reuniâso ondinária realizada no dis 05 de outubro de 2018 analisos o Próseto de Pesquisa acima cirada, considerando-o como APROVADO.

Lembramos que esbe ao pesquissdor elaborar e aprescritir a este Comite, relatcóios. parciais semestrais e final, de acardo com a Resoluçäa n $466 / 2012$ do Conselho Nacional de Sexide, inciso XI.2, letra "d".

O primeiro relatório está previsto para 05 de ahril de 2019.

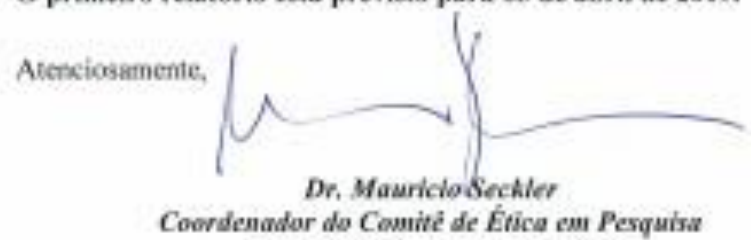

Hospival Univenintrio do USP 